IIE Transactions

\title{
Dynamic lot sizing problem for a warm/cold process
}

\section{Ayhan Özgür Toy \& Emre Berk}

To cite this article: Ayhan Özgür Toy \& Emre Berk (2006) Dynamic lot sizing problem for a warm/ cold process, IIE Transactions, 38:11, 1027-1044, DOI: 10.1080/07408170600854511

To link to this article: http://dx.doi.org/10.1080/07408170600854511

册 Published online: 23 Feb 2007.

Submit your article to this journal $[\pi$

山 Article views: 80

Q View related articles $\sqsubset$

Citing articles: 8 View citing articles 둔 


\title{
Dynamic lot sizing problem for a warm/cold process
}

\author{
AYHAN ÖZGÜR TOY and EMRE BERK* \\ Faculty of Business Administration, Bilkent University, 06800 Ankara, Turkey \\ E-mail: eberk@bilkent.edu.tr
}

Received April 2004 and accepted June 2005

\begin{abstract}
We consider a dynamic lot sizing problem with finite capacity for a process that can be kept warm until the next production period at a unit variable cost $\omega_{t}$ only if more than a threshold value has been produced and is cold, otherwise. That is, the setup cost in period $t$ is $K_{t}$ if $x_{t-1}<Q_{t-1}$ and $k_{t}$, otherwise $\left(0 \leq k_{t} \leq K_{t}\right)$. We develop a dynamic programming formulation of the problem, establish theoretical results on the structure of the optimal production plan and discuss its computational complexity in the presence of Wagner-Whitin-type cost structures. Based on our stuctural results, we present an optimal polynomial-time solution algorithm for $k_{t}=0$, and also show that an optimal linear-time solution algorithm exists for a special case. Our numerical study indicates that utilizing the undertime option (i.e., keeping the process warm via reduced production rates) results in significant cost savings, which has managerial implications for capacity planning and selection.
\end{abstract}

\section{Introduction}

Inventory replenishment processes, whether they consist of direct production or purchasing in a supply chain, typically involve setups. In a manufacturing setting, a setup is a set of operations to prepare for production that can include activities such as cleaning, warming up and calibrating equipment, and readying the shop floor and workforce. In a purchasing setting, the fixed set of activities performed to expedite an order can include the identification of suppliers, legal and clerical documentation, customs clearance of imports, shipment of goods, inspection of incoming goods, unloading etc. Associated with each of these activities, an out-of-pocket setup cost may be incurred. The dynamic lot sizing problem is the management of such a replenishment process by determining the production (purchasing) plan which minimizes the total setup, production (purchasing) and holding costs for an inventorable item, facing known demands over a finite number of time periods.

In some cases, it may be possible to avoid some of the activities typically included in a setup by keeping the process "warm" until the start of the next time period. Then, a smaller portion of the set of setup activities (such as only cleaning), if any, are performed at the beginning of the next period. Thus, one can speak of a major setup, which involves the original set of preparative activities, for a cold process and a minor setup, which involves a smaller subset of preparative activities, for a warm process. For example Agra and Constantino (1999) consider a single-item setting

${ }^{*}$ Corresponding author in which a minor setup cost is incurred if the process is ready (i.e., if it was set up for production in the previous period) and a major setup cost, otherwise. In their formulation, it is assumed that if a setup is performed for the item in a period, the process will be ready for use in the following period regardless of the quantity produced. However, as we discuss below, this may not be feasible and/or desirable in certain production/replenishment environments. In this paper, we consider a dynamic lot sizing problem with finite capacity in which the process can be kept warm for the next period only if a minimum amount has been produced and is cold, otherwise. The lot sizing problem setting that we investigate is encountered in a number of environments. Process industries such as glass, steel and ceramic production provide the leading examples of cases in which the physical nature of the production processes dictates that the processes be literally kept warm in certain periods to avoid expensive shutdown/startups. A particularly striking example with which the authors are familiar comes from the glass industry; in some periods, the glass production is continued in order to avoid substantial shutdown/startup costs but the produced glass is deliberately broken on the production line and fed back into the furnace! In this case, the process is being kept warm at the additional cost of breakage (plus some costs for non-reusable materials consumed). Similar practices are used in foundries; ceramic and brick ovens are also kept warm sometimes even though no further production is done in the current period to avoid costly cooling and reheating procedures. Aside from such literal manifestations, a process can also be kept warm in an abstract sense. Robinson and Sahin (2001) cite specific examples from the food and petrochemical industries where certain clean up and 
inspection operations can be avoided in the next period if the quantity produced in the current period exceeds a certain threshold (that is, the current production continues until the next period). This may be done through either overtime or undertime. The treatment of the overtime option is outside the scope of our analysis; however, deliberate undertime practices can be studied within our context of warm/cold processes. With undertime, processes can be kept warm by reducing the "nominal" or "calibrated" production rate within a prespecified range (e.g., Silver (1990), Moon et al. (1991) and Gallego (1993)). As an illustration, suppose that the process is capable of producing at most $R$ units at a nominal production rate in a certain time period. Furthermore, suppose that its production rate can be reduced so that, within the same time period, the process can produce $Q(<R)$ units at the slowest rate. Thus, it is possible to keep this process warm by having it operate at rates lower than nominal so long as the quantity to be produced is between $Q$ and $R$.

Such variable production rates are quite common in both process and discrete-item manufacturing industries since they both allow feeder mechanisms to be adjusted so as to set almost any pace to a line. For example, some chemical operations such as electroplating and fermentation can be decelerated deliberately (within certain bounds), and, manual operations can be slowed down by inserting idle times between units. Depending on the nature of the operations involved, the reduction in production rate can be obtained at either zero or positive additional cost. This additional variable cost is then the variable cost of keeping the process warm until the next period. Furthermore, if one can use the process for multiple purposes (e.g., different products) there may be additional variable costs due to keeping the process idle for the remainder of the current period (e.g., lost profit on other product(s) not produced). Then, the cost of keeping the process warm would also include idleness costs. Aside from direct economic calculations, a managerial decision on a warm process threshold may also be influenced by non-economic considerations such as: (i) the safety of mounted tools and fixtures left idle on the machinery; (ii) the impact on worker morale of engaging them in non-productive activities; and (iii) the impact learning/forgetting about phenomena on subsequent runs, etc. Hence, there may be managerially imposed policies in place that dictate the process be kept warm until the next period only if the production quantity in the current period exceeds a certain level, say, $Q$.

Another example of the setting we consider can be found in a replenishment environment where the supplier and/or shipper offers rebates that can be exercised in the next period if the amount ordered in the current period exceeds a certain quantity. In this procurement setting, the replenishment process is kept warm by ordering in quantities larger than a prespecified amount, say, $Q$, in a certain period. The additional cost of keeping the replenishment process warm until the next period is then zero for periods with ordering quantities larger than $Q$. Although such rebate structures would have a significant impact on the operational performance of supply chains via coordination and smoothing of orders between echelons, they have not received any attention in the literature. We believe that our proposed model provides a building block for the analysis and design of such two-party contracts. Note that the production processes cited above need to be modeled as capacitated, whereas, the replenishment processes may be uncapacitated.

As the above examples illustrate, the dynamic lot sizing problem in the presence of production-quantity-dependent warm/cold processes is a rather common problem. However, to the best of our knowledge, this problem has not been previously studied. Below, we briefly review related works in the vast literature on dynamic lot sizing.

The first formulation of the dynamic lot sizing problem was by Wagner and Whitin (1958) who assumed uncapacitated production and no shortages; this situation is generally labeled as the Wagner-Whitin problem. We shall henceforth refer to this problem and its setting as the "classical problem". Wagner and Whitin (1958) provided a dynamic programming solution algorithm and structural results on the optimal solution of the classical problem. Their fundamental contribution lies in the identification of planning horizons, which made forward solution algorithms possible. From the numerous other studies on the extension of the classical problem, we highlight these works that examine backordering and general cost structures (Zangwill, 1966; Blackburn and Kunreuther, 1974) and the Capacitated Lot Sizing Problem (CLSP) (Manne, 1958; Florian and Klein, 1971; Jagannathan and Rao, 1973; Love, 1973; Baker et al., 1978; Bitran and Matsuo, 1986; Bitran and Yanasse, 1982; Hindi; 1995a). For comprehensive reviews of the existing literature with more detailed taxonomies, we refer the reader to Aggarwal and Park (1993) and Wolsey (1995). Excellent surveys on reformulations and algorithms for the CLSP and uncapacitated lot sizing problems are provided in Pochet and Wolsey (1995) and Karimi (2003).

The works that are most closely related to ours are those that consider reserving a period for production with the option of not producing anything in that period. This setting also occurs as a subproblem of the multi-item CLSP with Lagrangean multipliers used as the reservation costs for each of the periods which has been studied by Eppen and Martin (1987), Karmarkar et al. (1987) Hindi (1995a, 1995b), and Agra and Constantino (1999). Although the models on lot sizing with reservation options apply the concept of a warm process, they do not consider a lower bound on the quantity produced for keeping the process warm until the next period. Thus, their results are not readily applicable to the setting with positive warm process thresholds that we consider. Similarly, we cannot rely on the results in the vast literature on multi-item CLSPs with sequence-dependent setups that consider warm processes but assume only warm process thresholds of zero production. (See Allahverdi et al. (1999) for an extensive review on this subject.) 
Another body of work that applies the concept of warm processes is contained in the literature on the Discrete Lot sizing and Scheduling Problem (DLSP) (e.g., Fleischmann (1990), Bruggemann and Jahnke (2000), and Loparic et al. (2003)). This body of work differs from ours in the use of a small-bucket approach (i.e., $R=1$ in every period) and, more importantly, in that the process can be kept warm only if there has been capacitated production in the current period (i.e., $Q=R$ in every period). Thus, the results in the DLSP literature are not readily applicable to our general setting.

Finally, we mention studies performed on lot sizing with undertime options. Silver (1990), Moon et al. (1991), and Gallego (1993) have examined the impact of using undertime via reduced production rates within the framework of a multiple-item lot scheduling problem with common cycles. Eiamkanchanalai and Banerjee (1999) also allow for bidirectional changes in the production rate. In all of the aforementioned studies, the analyses consider a constant demand rate with the objective of cost rate minimization under continuous review. Our model differs from these works in that our setting uses a periodic review over a finite horizon and has a single product with deterministic but variable demands. Due to the variable nature of demand, we do not obtain a single, stationary solution as in other works but rather establish the structure of the optimal production plan and conditions on the existence of forward solutions.

In this paper, we: (i) develop a dynamic programming formulation of the dynamic lot sizing problem for a warm/cold process; (ii) establish the structure of the optimal policy; (iii) show that polynomial-time and linear-time solution algorithms exist; and (iv) examine, via a numerical study, the sensitivity of the optimal production schedule and total cost to various system parameters and illustrate that restricting or ignoring the use of the undertime (warming) option results in substantial savings. To the best of our knowledge, this is the first work that considers warm/cold processes in the presence of warm process thresholds that depend on the production quantities in the previous period. We believe that our main contribution lies in establishing the structure of the optimal solution and proving a number of other properties of the dynamic lot sizing problem with warm process thresholds. Our numerical results also provide managerial insights into capacity selection decisions for warm/cold processes.

The rest of the paper is organized as follows: In Section 2 , we present the basic assumptions of our model and formulate the optimization problem. In Section 3, we provide theoretical results on the structure of the optimal solution. Finally, in Section 4, we discuss computational complexity issues and provide the findings of our numerical study. We also present, in the Appendix, an illustrative numerical example to highlight some key theoretical results, a forward dynamic programming solution algorithm and proofs of two of our major results.

\section{Model: Assumptions and Formulation}

We assume that the length of the problem horizon, $N$ is finite and known. The amount of demand in period $t$ is denoted by $D_{t}(t=1,2, \ldots, N)$. All demands are non-negative and known, but may be different over the problem horizon. No shortages are allowed; that is, the amount demanded in a period has to be produced in or before its period. The amount of production in period $t$ is denoted by $x_{t}$. For every item produced in period $t$, a unit production cost $c_{t}$ is incurred. The inventory on hand at the end of period $t$ is denoted by $y_{t}$; an inventory holding cost $h_{t}$ is incurred for every unit of ending inventory in the period. Without loss of generality, we assume that the initial inventory level is zero.

The production in a period is non-negative with a maximum capacity, $R_{t}$. We assume that physical capacities are non-decreasing, i.e., $R_{t-1} \leq R_{t}$ for all $t$ and make no assumption on the demand structure other than that $\sum_{i=t}^{j} D_{i} \leq \sum_{i=t}^{j} R_{i}$ for $1 \leq t \leq j \leq N$ for feasibility. We consider both warm and cold production processes. The production process may be kept warm until the beginning of period $t$ if $x_{t-1} \geq Q_{t-1}$; otherwise, the process cannot be kept warm and is cold. In order to keep the process warm until period $t, \omega_{t-1}$ is charged for every unit of unused capacity in period $t-1$. That is, the warming cost incurred in period $t-1$ would be $\omega_{t-1}\left(R_{t-1}-x_{t-1}\right)$ monetary units. Note that even if the quantity produced in period $t-1$ is at least $Q_{t-1}$, it may not be optimal to keep the process warm until the next period if during the next period, there would be no production activity; in such instances, there will be no warming costs incurred although $x_{t-1} \geq Q_{t-1}\left(\right.$ since $\left.x_{t}=0\right)$ ). A warm process requires a warm setup with an incurred cost $k_{t}$, and a cold process requires a cold setup with an incurred cost $K_{t}$, if production is to be done in period $t ; K_{t} \geq k_{t}$ for all $t$. We assume that all setup costs are non-negative, with $K_{t+1} \leq K_{t}$ and $k_{t+1} \leq k_{t}$ for all $t$. Furthermore, in the following, we assume $\max \left(0, \hat{Q}_{t}\right)<Q_{t} \leq R_{t}$ where $\hat{Q}_{t}$ denotes the point of indifference for a cold setup and is defined as $R_{t}-\left(\left(K_{t+1}-k_{t+1}\right) / \omega_{t}\right)$ for all $t$. (We discuss the consequences of relaxing this assumption in Section 3.1.)

Clearly, for $Q_{t}>R_{t}$ and $k_{t}=K_{t}$, we have the CLSP setting; and, as $Q_{t}\left(=R_{t}\right) \rightarrow \infty$, we get the classical problem setting.

The single-item CLSP with complex setup structures is known to be NP-hard (Bitran and Yanasse, 1982). Therefore, it is very difficult to optimally solve large instances of the problem. In fact, the solution time grows exponentially as the number of planning periods increase. However, for certain cost structures, it is possible to obtain analytical results on the structural and computational properties of the optimal production plan. Hence, we consider only the socalled Wagner-Whitin-type cost structures over the horizon of the problem. Specifically, we assume that $c_{t}+h_{t}>c_{t+1}$, $c_{t}+h_{t}-\omega_{t}>c_{t+1}, c_{t}+h_{t}-\omega_{t}>c_{t+1}-\omega_{t+1}$ for all $t$. 
This cost structure ensures that Wagner-Whitin-type costs are incurred for production levels that: (i) do not exceed the warm thresholds in either of the consecutive periods; (ii) exceed the threshold level in one period but not in the other period; and (iii) exceed the threshold level in both periods. Therefore, the above cost structure is essential for the results obtained in this paper.

The objective is to find a production schedule $x_{t} \geq 0(t=$ $1,2, \ldots, N)$ (timing and amount of production), such that all demands are met at minimum total cost. We develop a Dynamic Programming (DP) formulation of the problem

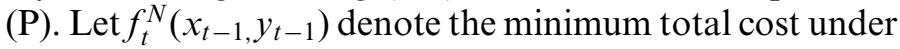
an optimal production schedule for periods $t$ through $N$, where $x_{t}$ is the production quantity and $y_{t-1}$ is the starting inventory for period $t$. Then

$(P)$ :

$$
\begin{aligned}
& f_{t}^{N}\left(x_{t-1}, y_{t-1}\right) \\
& =\min _{\substack{0 \leq x_{t} \leq R_{t} \\
x_{t}+y_{t} \geq 1 \geq D_{t}}}\left[\begin{array}{c}
K_{t} \times \delta_{t} \times z_{t} \\
+\left[k_{t}+\omega_{t-1}\left(R_{t-1}-x_{t-1}\right)\right] \times \delta_{t} \times\left(1-z_{t}\right) \\
+c_{t} \times x_{t}+h_{t} \times y_{t}+f_{t+1}^{N}\left(x_{t}, y_{t}\right)
\end{array}\right],
\end{aligned}
$$

where

$$
\begin{gathered}
y_{t}=y_{t-1}+x_{t}-D_{t} \quad \text { for } t=1,2, \ldots, N, \\
\delta_{t}=\left\{\begin{array}{ll}
0 & \text { if } x_{t}=0 \\
1 & \text { if } x_{t}>0
\end{array} \text { for } t=1,2, \ldots, N,\right.
\end{gathered}
$$

and

$$
z_{t+1}=\left\{\begin{array}{ll}
0 & \text { if } x_{t} \geq Q_{t} \\
1 & \text { if } x_{t}<Q_{t}
\end{array} \quad \text { for } t=1,2, \ldots, N-1,\right.
$$

with the boundary condition in period $N$ being:

$$
\begin{aligned}
& f_{N}^{N}\left(x_{N-1}, y_{N-1}\right) \\
& =\min _{\substack{0 \leq x_{N} \leq R_{N} \\
x_{N}+y_{N-1} \geq D_{N}}}\left[\begin{array}{c}
K_{N} \times \delta_{N} \times z_{N} \\
+\left[k_{N}+\omega_{N-1}\left(R_{N-1}-x_{N-1}\right)\right] \times \delta_{N} \times\left(1-z_{N}\right) \\
+c_{N} \times x_{N}+h_{N} \times y_{N}
\end{array}\right] .
\end{aligned}
$$

The optimal solution is found using the above recurrence and $f_{1}^{N}(0,0)$ denotes the minimum cost of supplying the demand for periods 1 through $N$ (where we arbitrarily set $x_{t-1}=0$ ). We are now ready to examine some of the structural properties of the optimal solution to the above formulation. (Without loss of generality we assume throughout that $y_{0}=y_{N}=0$ and, for convenience, $R_{0}=\omega_{0}=0$.)

\section{Structural results}

In this section, we present structural results on the optimal production plan for the lot sizing problem with a warm/cold process. In particular, we establish the conditions under which production is to be done and the amount of production in a period. Furthermore, we show that certain production plans enable one to partition the original problem into independently solvable subproblems.

First, we provide an equivalence property which will simplify our development of further structural results.

Proposition 1. If problem $(P)$ is feasible, it can be written as an equivalent CLSP where in each period the demand is not greater than the capacity.

Proof. A proof is provided in the Appendix.

Therefore, without loss of generality, we shall assume in the following that $D_{t} \leq R_{t}$ for all $t$; this, naturally, ensures the feasibility condition. An important property that plays a key role in developing algorithms to solve lot sizing problems is the one that states when to do a setup and to produce. In the absence of warm/cold processes, Bitran and Yanasse (1982) provide a property of the optimal solution which states that, for capacitated settings where, over the horizon, no prespecified pattern exists for setup costs, unit holding costs and capacities, and unit production costs are non-increasing $(G / G / N I / G$ setting in their notation): production is done in a period only if there is insufficient inventory to satisfy the demand for that period (Proposition 2.4 in Bitran and Yanasse (1982)). In the presence of warm/cold processes, this property no longer holds. Below, we present an extension of their result to the instance where there are quantity-dependent warm processes.

Theorem 1. An optimal production plan has the property $z_{t} \times$ $x_{t} \times\left[y_{t-1}-D_{t}\right]^{+}=0$ for $t=1,2, \ldots, N$ where $z_{t}, x_{t}$ and $y_{t-1}$ are as given in Equations (2)-(4).

Proof. A proof is provided in the Appendix.

As expected, Theorem 1 reduces to Proposition 2.4 in Bitran and Yanasse (1982) when $k_{t}=K_{t}$ (i.e., $z_{t}=1$ ) for all $t$. In the presence of warm/cold processes, however, we see that it may be optimal to produce even in a period of zero demand, which is not the case for the classical setting (see Corollary 2.1 in Bitran and Yanasse (1982)).

In the classical problem setting, it is established that, in an optimal production plan, the values that the production quantities can take on in any period are either zero or exactly equal to a sum of demands for a finite number of periods into the future. In the CLSP, however, the optimal production plan is composed of subplans in which the production quantities in any period are either zero or at capacity, except for at most one period in which it is less than capacity. In the presence of quantity-dependent warm/cold processes, these fundamental results no longer hold in general for all periods. In the following, we establish certain structural properties of the optimal plans and gradually develop the structure of the optimal solution for the CLSP with quantity-dependent warm/cold processes.

We introduce the following definitions. Let $X=$ $\left\{x_{1}, \ldots, x_{N}\right\}$ denote a feasible production plan constructed over periods 1 through $N$ and, let period $t$ be called a 
regeneration point if $y_{t-1}=0, z_{t}=1$ and $x_{t}>0$. Also, let $\Psi_{u v \mid I_{u-1}, I_{v-1}}$ represent a subset of $X$ between two consecutive cold setups $u$ and $v$ with given starting and ending inventories given such that $y_{u-1}=I_{u-1}$ and $y_{v-1}=I_{v-1}$. We have $\Psi_{u v \mid I_{u-1}, I_{v-1}}=\left\{x_{i} \mid x_{i}>0, i=u, \ldots, m ; x_{i}=0\right.$ for $i=m+1, \ldots, v-1 ; z_{u}=1=z_{v} ; z_{i}=0$ for $u+1 \leq i \leq m$; $z_{m+1} \geq 0$ and $z_{i}=1$ for $\left.m+2 \leq i \leq v-1\right\}$ where $m$ denotes the latest period in which production is done between $u$ and $v-1$ for $0 \leq u \leq m<v \leq N+1$.

We will call $\Psi_{u v \mid I_{u-1}, I_{v-1}}$ a production series, and a period $t$ for $u+1 \leq t \leq m-1$ will be called an intermediate production period. Note that, a production series may begin and end with positive inventory, i.e., $I_{u-1} \geq 0, I_{v-1} \geq 0$. Therefore, the first period of a production series is not necessarily a regeneration point as defined above. However, from Theorem 1 , for cold setups to exist in periods $u$ and $v$, we must have $I_{t}<D_{t+1}$ for $t=u-1, v-1$. (It is possible to form feasible series which violate this condition, but they may safely be ignored due to their suboptimality.) By using the following result, we will further simplify our development and, henceforth, consider only production series that have zero starting and ending inventories.

Proposition 2. If $\Psi_{u v \mid I_{u-1}, I_{v-1}}$ (with $I_{u-1}<D_{u}$ and $I_{v-1}<D_{v}$ ) is a subset of an optimal plan for problem $(\mathrm{P})$ with demands $D_{t}$ over periods $u$ through $v-1$, then $\Psi_{u v \mid 0,0}^{\prime}$, which has the same production schedule, is a subset of an optimal plan for problem $\left(\mathrm{P}^{\prime}\right)$ with demands $D_{u}^{\prime}=D_{u}-I_{u-1}$ and $D_{v-1}^{\prime}=D_{v-1}+I_{v-1}$ ceteris paribus.

Proof. A proof is provided in the Appendix.

It follows from above that a series denoted by $\Psi_{u v \mid I_{u-1}, I_{v-1}}$ can be substituted by $\Psi_{u v \mid 0,0}$, which we shorten to $\Psi_{u v}$. Feasibility of a production series implies that the physical capacity constraint and no backordering assumption are not violated. Hence, in the optimal plan $\left[D_{i}-y_{i-1}\right]^{+} \leq x_{i} \leq R_{i}$ for $u \leq i \leq m$. Furthermore, from the definition of a warm setup one intuitively obtains $x_{i} \geq Q_{i}$ for $u \leq i \leq m-1$. Thus, for any optimal series, $\max \left(Q_{i},\left[D_{i}-y_{i-1}\right]^{+}\right) \leq x_{i} \leq$ $R_{i}$ for $u \leq i \leq m-1$.

For exposition purposes, we make a distinction between two instances of production at capacity. We shall refer to the production instance $x_{i}=R_{i}$ as capacitated production only if $\left[D_{i}-y_{i-1}\right]^{+}<R_{i}$. Hence, $x_{i}=\left[D_{i}-y_{i-1}\right]^{+}=R_{i}$ will not be referred to as capacitated production but will simply be called production at capacity. (As it will become clear, we make this distinction to identify the successive capacitated periods which emerge from/are found in the end of a production series.)

As we establish in the following lemma, in addition to the physical capacity in period $t$, there is also an economic bound, $E_{t}$ on the production quantity in the presence of warm/cold processes. That is, $E_{t}$ is such a quantity that, producing more than this quantity in period $t$ for a future period, is more costly than producing the excess quantity in period $t+1$.
Lemma 1. In an optimal production plan:

(i) $x_{t} \leq E_{t}$ where,

$\begin{aligned} E_{t}= & \frac{\max \left(Q_{t},\left[D_{t}-y_{t-1}\right]^{+}\right)\left(c_{t}+h_{t}-c_{t+1}-\omega_{t}\right)+k_{t+1}+R_{t} \omega_{t}}{c_{t}+h_{t}-c_{t+1}} \\ & \times \text { for } \forall t .\end{aligned}$

(ii) $x_{t}=R_{t}$ only if $E_{t} \geq R_{t}$.

Proof. A proof is provided in the Appendix.

Lemma 2. A production series $\Psi_{u v}$, in which there is at least one period $t$ such that $x_{t}=R_{t}\left(>Q_{t}\right), 0<x_{t+1}<R_{t+1}$, and $y_{t}>0$, cannot be optimal.

Proof. A proof is provided in the Appendix.

From Lemmas 1 and 2, we get the following corollary.

Corollary 1. In a production series (of an optimal production plan) in which $m$ denotes the last period of production in the series:

(i) If $x_{t}=R_{t}\left(>Q_{t}\right)$ and $0<x_{t+1}<R_{t+1}$ then $y_{t}=0$ for $u \leq t \leq m-1$.

(ii) If $x_{t}=R_{t}\left(>Q_{t}\right)$ and $y_{t}>0$ then $x_{t+1}=R_{t+1}$ for $u \leq$ $t \leq m-1$.

(iii) Let $m-r+1$ denote the first period in which capacitated production is done in a production series of an optimal plan. Then, $m-r+1 \geq \max \left(j \mid E_{j}<R_{j}\right.$ for $u \leq$ $j \leq m)$ and $x_{t}=R_{t}$ for $m-r+1 \leq t \leq m$.

The above corollary provides the basis of the subtle distinction we like to make between "production at capacity" and "capacitated production". The first refers to the case where production at capacity is done solely to satisfy the net demand of the period (i.e., $y_{t}=0$ ) in part (i), whereas the latter refers to production again at capacity but to satisfy more than the net demand in that period (i.e., $y_{t}>0$ ) in part (ii). Corollary 1 also implies that, if there is a succession of capacitated production periods, the last capacitated production period coincides with the last production period in the series of an optimal production plan! This result is important in that it gives us the structure in which an optimal production series ends.

Lemma 3. In an optimal production series $\Psi_{u v},($ for $u \leq t \leq$ $m-1), x_{t}>\max \left(Q_{t},\left[D_{t}-y_{t-1}\right]^{+}\right)$only if $x_{t+1}=R_{t+1}$ and $y_{t+1}>0$.

Proof. A proof is provided in the Appendix.

Corollary 2. In a production series $\Psi_{u v}$ (of an optimal production plan) in which production is done last in period $m$, if $x_{m}<R_{m}$ then $x_{t}=R_{t}\left(>Q_{t}\right)$ only if $y_{t}=0$ for $u \leq t \leq$ $m-1$.

We are now in a position to provide the structure of a production series in an optimal production plan.

Theorem 2. (The optimal production quantity theorem.) In a production series $\Psi_{u v}$ of an optimal production plan, in which 
$m$ is the last period in which production is done and $r(\geq 0)$ is the number of successive periods with capacitated production:

(i) $x_{t}=\max \left(Q_{t},\left[D_{t}-y_{t-1}\right]^{+}\right)$, for $u \leq t \leq m-r-1$ and $m-u>r \geq 0$.

(ii) $x_{m-r}=\left[\sum_{i=m-r}^{v-1} D_{i}-y_{m-r-1}-\sum_{i=m-r+1}^{m} R_{i}\right]^{+}<$ $\min \left(E_{m-r}, R_{m-r}\right)$ and $m-u \geq r \geq 0$.

(iii) $x_{t}=R_{t}\left(\leq E_{t}\right)$ for $m-r+1 \leq t \leq m$ and $r \geq 1$.

Proof. A proof is provided in the Appendix.

Theorem 2 gives the values that production quantities in any period may assume in an optimal production plan in the presence of quantity-dependent warm/cold processes. The above theorem gives, as special cases, the results in Wagner and Whitin (1958, Theorem 2, p. 91) when $Q_{t}\left(=R_{t}\right) \rightarrow \infty$, and those in Florian and Klein (1971, Corollary, p. 16) when $Q_{t}=R_{t}=R$ and $k_{t}=K_{t}$, for all $t$. Thus, it enables one to identify the forms of the production series to be considered in solving problem $(\mathrm{P})$ and forms the basis of the solution algorithms we develop in a later section. To that end, we provide the following corollary.

Corollary 3. In an optimal production plan, the series $\Psi_{u v}$ can only have the following forms:

(i) $x_{u}=\sum_{i=u}^{v-1} D_{i}, x_{i}=0$ for $u+1 \leq i \leq v-1$.

(ii) $x_{i}=\max \left(Q_{t},\left[D_{t}-y_{t-1}\right]^{+}\right)$for $u \leq i \leq m-1$ $x_{m}=\sum_{i=m}^{v-1} D_{i}-y_{m-1}, x_{i}=0$ for $m+1 \leq i \leq v-1$.

(iii) $x_{i}=R_{i}$ for $u \leq i \leq m, x_{i}=0$ for $m+1 \leq i \leq v-1$.

(iv) $x_{u}=\left[\sum_{i=m-r}^{v-1} D_{i}-y_{m-r-1}-\sum_{i=m-r+1}^{m} R_{i}\right]^{+}, x_{i}=R_{i}$ for $u+1 \leq i \leq m, x_{i}=0$ for $m+1 \leq i \leq v-1$.

(v) $x_{i}=\max \left(Q_{t},\left[D_{t}-y_{t-1}\right]^{+}\right)$for $u \leq i \leq m-r-1$, $x_{m-r}=\left[\sum_{i=m-r}^{v-1} D_{i}-y_{m-r-1}-\sum_{i=m-r+1}^{m} R_{i}\right]^{+}, \quad x_{i}=$ $R_{i}$ for $m-r+1 \leq i \leq m, \quad x_{i}=0$ for $m+1 \leq i \leq$ $v-1$.

Maintaining the definition of a regeneration point given above, let $S_{u v}$ denote a subset of a feasible production plan $X$ such that $S_{u v}$ includes the components of $X$ for all periods between the two consecutive regeneration points $u$ and $v$; that is, $S_{u v}=\left\{x_{i}, i=u, \ldots, v-1 \mid z_{u}=1=z_{v}\right.$ and $y_{u-1}=$ $0=y_{v-1} ; y_{i} \geq 0$ for $\left.u<i<v\right\}$ where $1 \leq u<v \leq N+1$. We will refer to $S_{u v}$ as a production sequence. Clearly, any feasible production plan is composed of one or more production sequences and since $y_{0}=y_{N}=0$, at least one production sequence exists in an $N$-period problem. Moreover, any production sequence is composed of at least one production series.

In the CLSP setting, a capacity constrained production sequence is defined in Florian and Klein (1971) as a production sequence in which the production level of at most one period is positive but less than capacity, and all other productions are either zero or at their capacity. In the presence of warm/cold processes, we define a capacity-constrained production series as a production series in which all productions are either zero or at their capacity. That is, we accept only the series of the form given in Corollary 3 part (iii) as a capacity-constrained series.

\section{Theorem 3. (Capacity-constrained series theorem.)}

(i) In the presence of warm/cold processes, an optimal production plan consists of production sequences in which at most one series is not a capacity-constrained production series.

(ii) Moreover, if there exists a series which is not capacity constrained, then, it is the first series of that sequence.

Proof. A proof is provided in the Appendix.

Corollary 4. An optimal production plan has the property $z_{t} \times y_{t-1} \times x_{t} \times\left(R_{t}-x_{t}\right)=0$ for $t=1,2, \ldots, N$ where $z_{t}$, $x_{t}$ and $y_{t-1}$ are as given in Equations (2)-(4).

Proof. A proof is provided in the Appendix.

Note that the above result is another extension of the result on $G / G / N I / G$ (in the notation of the Bitran-Yanasse) CLSP with warm/cold processes.

A case of theoretical interest and practical significance is when $k_{t}=0$. This corresponds to a production setting where setup carry-over is possible at no cost. For example, in glass manufacturing, keeping the furnaces warm essentially ensures that production in the next period starts with no setup. Other practical applications include a production line whose physical layout or a machine whose calibration is maintained for the next period at no or almost no additional fixed cost.

Theorem 4. (Single-series theorem.) When $k_{t}=0 \quad \forall t=$ $1,2, \ldots, N$, than:

(i) Each sequence $S_{u v}$ comprises only one production series $\Psi_{u v}$. This series is of the form:

(a) $x_{t}=\max \left(Q_{t},\left[D_{t}-y_{t-1}\right]^{+}\right)$, for $u \leq t \leq m-1$ and $m-u>0$

(b) $x_{m}=\varepsilon$, where $\varepsilon=\left[\sum_{i=m}^{v-1} D_{i}-y_{m-1}\right]^{+}<R_{m}$ and $m-$ $u \geq 0$.

(ii) An optimal production plan has the property $z_{t} \times x_{t} \times$ $y_{t-1}=0$ where $z_{t}, x_{t}, y_{t-1}$ are as given in Equations (2)(4).

Proof. A proof is provided in the Appendix.

Another useful property in solving lot sizing problems is that of partition. In the absence of warm/cold processes, it is possible to optimally partition a longer problem if the constraint $y_{t}=0$ is imposed in a period within the horizon for both the classical problem and the CLSP while ensuring capacity feasibility for the remainder of the decomposed problem (Florian and Klein, 1971). In the presence of quantity-dependent warm/cold processes, however, the state of the system is no longer fully represented by the current inventory level in a period and further conditions are needed for a partition. 
In the following theorem, we state such conditions.

Theorem 5. (Partition theorem.) Suppose that $k_{j}=0$ for $\forall j$. then:

(i) If $y_{t-1}=0$ and $x_{t-1}<Q_{t-1}$ in a $t$-period problem then it is optimal to consider periods 1 through $t-1$ by themselves in any feasible $t^{*}$ - period problem $\left(t^{*} \geq t\right)$; that is, a cold partition occurs in period $t$.

(ii) If $x_{t-1} \geq Q_{t-1}, y_{t-1}=0, x_{t}>0$ and $E_{t}<R_{t}$ in a $t-$ period problem, then it is optimal to consider periods 1 through $t-1$ by themselves in any feasible $t^{*}$-period problem $\left(t^{*} \geq t\right)$; that is, a warm partition occurs in period $t$.

Proof. A proof is provided in the Appendix.

Note that a partition condition exists only for the case for which the warm setup cost is zero. Otherwise, as the horizon of the problem is extended, it is possible to encounter optimal solutions that modify the production schedules in periods 1 through $t$ even if the above stated conditions hold. The existence of a partition implied in Theorem 5 is very important in that it also implies the existence of a forward solution algorithm. In Section 4.1, we elaborate more on such algorithms.

\subsection{A digression: If $Q_{t}<\hat{Q}_{t}$}

The structural results presented so far are based on the assumption that $Q_{t} \geq \hat{Q}_{t}, \forall t$, where $\hat{Q}_{t}$, defined as $R_{t}-$ $\left(K_{t+1}-k_{t+1}\right) / \omega_{t}$, represents the point of indifference between the costs of keeping the process warm until the next period and of incurring a cold setup in the next period. As the discussion below reveals, this is the most realistic setting. However, for completeness, we discuss the consequences of relaxing this assumption. When $Q_{t}<\hat{Q}_{t}$, for production quantities such that $Q_{t} \leq x_{t}<\hat{Q}_{t}$, the cost of keeping the process warm until the next period is $\left(R_{t}-x_{t}\right) \omega_{t}$. Since $x_{t}<\hat{Q}_{t}$, we have $\left(R_{t}-x_{t}\right) \omega_{t}>K_{t+1}-k_{t+1}$, which implies that keeping the system warm in this period yields a cost higher than that incurred by having a cold setup in the next period. Hence, when the managerially selected value of the warm process threshold is below $\hat{Q}_{t}$, the DP formulation (P) provided by Equation (1) subject to Equations (2)-(4) does not reveal the optimal schedule and the cost. This is mainly due to Equation (4) which is constructed under the assumption that $Q_{t} \geq \hat{Q}_{t}$. In the case where $Q_{t}<\hat{Q}_{t}$, a new DP formulation must include the warm process indicator $z_{t}$ as a binary decision variable, and, as such, the state of the system must be redefined to also include $z_{t}$. Even though it would be possible to reconstruct the DP formulation, it is easy to see that, in an optimal solution, no warming would be done if the production quantity is less than $\hat{Q}_{t}$ regardless of the value of the managerially set warm process threshold. Therefore, $\hat{Q}_{t}$ acts as a bound on the warm process decision. Therefore, it is possible to slightly modify the formulation provided in Equation (1) subject to Equations (2)-(4) to allow for arbitrarily set warm process thresholds by redefining $Q_{t}$ used in our formulation such that $Q_{t}=\max \left(\bar{Q}_{t}, \hat{Q}_{t}\right)$, where $\bar{Q}_{t}$ denotes the warm process threshold arbitrarily set by the management. The DP formulation (P) can then be used as it stands.

From the above arguments, it also follows that $\hat{Q}_{t}$ is the threshold value which gives the lowest possible cost for a given problem setting. Hence, if the management is free to choose the warm process threshold, it would always set it at the point of indifference. This observation is validated in our numerical studies.

An illustrative numerical example highlighting the key features of the optimal solution series and some other key results presented above are provided in the Appendix.

\section{Computational results}

\subsection{Solution algorithms}

The optimal solution to problem (P) can, theoretically, be obtained by a backward solution algorithm. However, in a backward solution algorithm, the state of the system needs to be described by the number of periods in the horizon, the ending inventory and production quantity in the previous period, and the maximum of the capacity and the total demand for the remainder of the horizon for each period. Even for discrete demand or largely discretized continuous demand scenarios, the size of the state space for reasonable problem settings becomes prohibitively high. Therefore, it is essential to develop forward solution algorithms when available.

For zero warm setup costs, $\left(k_{t}=0 \forall t\right)$, by invoking Theorems 3 and 4, one can obtain a forward DP solution algorithm which provides an optimal solution in polynomial time. In the Appendix, we provide such an algorithm. (Prior to using the suggested solution algorithm, we assume that the individual demands are smoothed to ensure feasibility of the problem, which can be done in $O(N)$.) For the computational complexity of the proposed algorithm, we provide the following brief discussion. For any given horizon length $T$, one generates $T$ subproblems such that the problem over the periods 1 through $T$ with $y_{T}=0,\left(\mathrm{P}_{1, T}\right)$, can be solved by decomposing as $\mathrm{P}_{1, T}=\mathrm{P}_{1, t}+\mathrm{P}_{t+1, T}$ with the imposed constraint that $y_{t}=0$ for $1 \leq t<T$. Thus, for problem (P), one needs to solve a total of $N(N+1) / 2$ subproblems. Each of these subproblems can be solved in $O(N)$ time. Hence, the algorithm provides an optimal solution in $O\left(N^{3}\right)$ time. The numerical study was conducted via this algorithm.

With additional conditions, it may also be possible to obtain solution algorithms with less complexity. In the following theorem, we state that an improved $O(N)$-time solution algorithm exists for one such special case. 
Theorem 6. (Improved $O(N)$ solution algorithm theorem.) Given an N-period instance of the dynamic lot sizing problem with warm/cold processes such that: $D_{t}<Q_{t}, k_{t}=$ 0 (thereby, $R_{t} \geq E_{t}=\max \left(Q_{t},\left[D_{t}-y_{t-1}\right]^{+}\right)=Q_{t}$ ) and $\omega_{t}=0$ for $1 \leq t \leq N$, an optimal production schedule can be found in $O(N)$ time.

Proof. Following Aggarwal and Park (1993), let $V(j)$ denote the minimum cost of supplying the demands of periods 1 through $j-1$ such that $y_{j-1}$ is zero for $1<j \leq N+1$ and $V(1)=0$. This definition implies that $V(N+1)$ is the cost of the optimal production plan for periods 1 through $N$. Now, consider a production sequence $S_{i j}=\left\{x_{i}=Q_{i}, x_{i+1}=Q_{i+1}, \ldots, x_{m-1}=\right.$ $\left.Q_{m-1}, x_{m}=\left(\sum_{t=i}^{j} D_{t}-\sum_{t=i}^{m-1} Q_{t}\right), x_{m+1}=0, \ldots, x_{j}=0\right\}$. Note that the optimality of such a sequence follows from Theorem 2. For $D_{t}<Q_{t}(t=1, \ldots, N)$, define the $N \times(N+1)$ array $\mathbf{A}=\{a[i, j]\}$, where $a[i, j]=V(i)+K_{i}+$ $\sum_{t=i+1}^{j} \eta_{t} k_{t}+\sum_{t=i}^{j} c_{t} x_{t}+\sum_{t=i}^{j} h_{t}\left(\sum_{j=1}^{t} x_{j}-\sum_{j=1}^{t} D_{j}\right)$ if $i<j$ and $+\infty$, otherwise. Following Theorem 2, we have $x_{t}=\eta_{t} \max \left\{\left(D_{t}-y_{t-1}\right), \min \left\{Q_{t}, \sum_{u=t}^{j} D_{u}-y_{t-1}\right\}\right\} \quad$ with $\eta_{t}=1$ if $\sum_{u=t}^{j} D_{u}-y_{t-1}>0$ and 0 , otherwise. Then, for $1<j \leq N+1, \quad V(j)=\min _{1 \leq i \leq n} a[i, j]$ if $D_{j-1}>0$ and, $V(j-1)$ otherwise.

Definition 1. After Aggarwal and Park (1993) (p. 556), an $p \times q$ two-dimensional array $\mathbf{A}=\{a[i, j]\}$ is Monge if for $1 \leq i<p$ and $1 \leq j<q$ :

$$
a[i+1, j+1]-a[i+1, j] \leq a[i, j+1]-a[i, j] .
$$

Consider the production sequence $S_{i j}=\left\{x_{i}=Q_{i}, x_{i+1}=\right.$ $Q_{i+1}, \ldots, x_{m-1}=Q_{m-1}, x_{m}=\left(\sum_{t=i}^{j} D_{t}-\sum_{t=i}^{m-1} Q_{t}\right), x_{m+1}=$ $\left.0, \ldots, x_{j}=0\right\}$. The given production sequence will result in a total cost of $a[i, j]$ as defined in Equation (6). Clearly, if a new period $(j+1)$ is added to the horizon, the quantity to satisfy some or all of its demand $D_{j+1}$ can at the earliest be produced in period $m$; and, the portion of the production sequence up to $m-1$ remains unchanged. Then, $a[i, j+1]$ is the sum of $a[i, j]$ and the costs of producing $D_{j+1}$ units starting from period $m$ and carrying them in inventory until period $j+1$. That is, the cost difference as a new period is added is only due to the production and holding costs incurred for the quantity to satisfy $D_{j+1}$. Now, consider the same demand pattern from period $i+1$ to period $j$. The production sequence in this case will be the same for periods $i+1$ through $m-1$, and the quantity $Q_{i}-D_{i}$, which was produced in period $i$ previously will now be produced in period $m$ (and in later periods if necessary). Let $m^{\prime}(\geq m)$ denote the latest period in which production is done for the production sequence starting in period $i+1$. Then, if a new period $(j+1)$ is added to the horizon, some or all of its demand $D_{j+1}$ can at the earliest be produced in period $m^{\prime}$; and, the portion of the production sequence up to period $m^{\prime}-1$ remains unchanged. The total cost of production $a[i+1, j+1]$ is the sum of $a[i+1, j]$ and the costs of producing $D_{j+1}$ units starting from period $m^{\prime}$ and carrying them in inventory until period $j+1$. Again, the cost difference as a new period is added is only due to the production and holding costs incurred for the quantity to satisfy $D_{j+1}$. We see that the increase in the total costs for a production sequence to satisfy demands over a given horizon as a new period $j+1$ is added to the horizon is equal to the production and holding costs of the quantity to satisfy the demand in period $j+1$. Due to the marginal production cost structure imposed, the production cost of any quantity to satisfy a demand in the future decreases as the quantity is produced in periods closer to the demand period. The holding cost decreases as well, since the number of periods over which inventory is held decreases. Therefore, $a[i, j+1]-a[i, j] \geq a[i+1, j+1]-a[i+1, j]$. Hence, we establish the Mongité of A given below:

Lemma 4. A is Monge if $D_{t} \leq Q_{t}, k_{t}=0$ and $\omega_{t}=0$ for $t=1,2, \ldots, N$.

Proof. Given the Mongité of $\mathbf{A}$ and linear preprocessing time, we can apply Eppstein's on-line array-searching algorithm (Eppstein, 1990). Hence, we have Theorem 6.

Following the arguments presented in the proof, note that for positive $k_{t}$ values, there may be additional cost reductions when period $j+1$ is added if $x_{m}>Q_{m}$ and/or $x_{m^{\prime}}>Q_{m^{\prime}}$ which implies that some of the production may be pushed forward. Hence, the Monge condition for A may no longer hold. Likewise, if $D_{t} \geq Q_{t}$ for some $t$, then we no longer have a two-dimensional array to define the costs since it is not guaranteed that $a[i, j]$ will always involve a cold setup as assumed in the above formulation. It may be interesting for future work to investigate similar linear search algorithms for this case using the properties of higher-dimensional Monge arrays (see Aggarwal and Park, 1989, 1993).

\subsection{Numerical study}

We conducted our numerical study to investigate three aspects: (i) the sensitivity of the optimal production schedule to various system parameters; (ii) the impact of managerial policies to keep processes warm; and (iii) optimal capacity determination in the presence of warm/cold processes.

For our numerical study, we considered a problem horizon of 100 periods. A base demand series was developed such that the base demand in period $t, D_{t}^{\text {base }}$, is either equal to zero with a probability of 0.20 or it is generated from the distribution $U(1,40)$ with a probability of 0.80 . We considered only integer demands in our analysis; hence, we truncated the generated random demand values to ensure integer values. Different tightness levels of the capacity were achieved by using six demand patterns as multiples of the base series (i.e., $D_{t}=M \times$ $D_{t}^{\text {base }}$ ). We considered constant parameters over the horizon of the problem; for all $t$, we set $h_{t}=h=1, k_{t}=0$, 
Table 1. First 25 periods of the optimal production schedules (medium demand, $R=100$ )

\begin{tabular}{|c|c|c|c|c|c|c|c|c|c|c|c|c|c|c|c|c|c|}
\hline \multirow[b]{3}{*}{$t$} & \multirow[b]{3}{*}{$D_{t}$} & \multicolumn{16}{|c|}{ Production level } \\
\hline & & \multicolumn{4}{|c|}{$w=0.05$} & \multicolumn{4}{|c|}{$w=0.55$} & \multicolumn{4}{|c|}{$w=0.95$} & \multicolumn{4}{|c|}{$Q=R$} \\
\hline & & $\begin{array}{l}K= \\
Q=\end{array}$ & $\begin{array}{c}75 \\
0\end{array}$ & $\begin{array}{c}50 \\
0\end{array}$ & $\begin{array}{c}25 \\
0\end{array}$ & $\begin{array}{l}K= \\
Q=\end{array}$ & $\begin{array}{c}75 \\
0\end{array}$ & $\begin{array}{c}50 \\
9.09\end{array}$ & $\begin{array}{c}25 \\
54.54\end{array}$ & $\begin{array}{l}K= \\
Q=\end{array}$ & $\begin{array}{c}75 \\
21.05\end{array}$ & $\begin{array}{c}50 \\
47.36\end{array}$ & $\begin{array}{c}25 \\
73.68\end{array}$ & $\begin{array}{l}K= \\
Q=\end{array}$ & $\begin{array}{c}75 \\
100\end{array}$ & $\begin{array}{c}50 \\
100\end{array}$ & $\begin{array}{c}25 \\
100\end{array}$ \\
\hline 1 & 25 & & 25 & 25 & 25 & & 25 & 52 & 25 & & 52 & 52 & 25 & & 52 & 52 & 25 \\
\hline 2 & 27 & & 27 & 27 & 27 & & 27 & & 27 & & & & 27 & & & & 27 \\
\hline 3 & 57 & & 57 & 57 & 57 & & 57 & 57 & 57 & & 57 & 57 & 57 & & 100 & 100 & 57 \\
\hline 4 & 92 & & 92 & 92 & 92 & & 92 & 94 & 94 & & 94 & 94 & 94 & & 51 & 51 & 94 \\
\hline 5 & 2 & & 2 & 2 & 2 & & 2 & & & & & & & & & & \\
\hline 6 & 80 & & 80 & 80 & 80 & & 80 & 80 & 80 & & 80 & 80 & 80 & & 100 & 100 & 80 \\
\hline 7 & 0 & & 0 & 0 & 0 & & 0 & 27 & & & 27 & 27 & & & 7 & 7 & \\
\hline 8 & 27 & & 27 & 27 & 27 & & 27 & & 27 & & & & 27 & & & & 27 \\
\hline 9 & 40 & & 40 & 40 & 40 & & 40 & 40 & 40 & & 75 & 75 & 40 & & 75 & 75 & 40 \\
\hline 10 & 20 & & 20 & 20 & 20 & & 20 & 35 & 35 & & & & 35 & & & & 35 \\
\hline 11 & 15 & & 15 & 15 & 15 & & 15 & & & & & & & & & & \\
\hline 12 & 42 & & 42 & 42 & 42 & & 42 & 42 & 42 & & 42 & 42 & 42 & & 100 & 42 & 42 \\
\hline 13 & 45 & & 45 & 45 & 45 & & 45 & 45 & 65 & & 87 & 65 & 65 & & 7 & 65 & 65 \\
\hline 14 & 20 & & 20 & 20 & 20 & & 20 & 42 & & & & & & & & & \\
\hline 15 & 22 & & 22 & 22 & 22 & & 22 & & 22 & & & 64 & 22 & & 64 & 64 & 22 \\
\hline 16 & 42 & & 42 & 42 & 42 & & 42 & 42 & 42 & & 42 & & 42 & & & & 42 \\
\hline 17 & 92 & & 92 & 92 & 92 & & 92 & 92 & 92 & & 92 & 92 & 92 & & 100 & 100 & 100 \\
\hline 18 & 42 & & 42 & 42 & 42 & & 42 & 42 & 42 & & 42 & 84 & 42 & & 76 & 76 & 34 \\
\hline 19 & 42 & & 42 & 42 & 42 & & 42 & 42 & 42 & & 42 & & 42 & & & & 42 \\
\hline 20 & 77 & & 77 & 77 & 77 & & 77 & 77 & 77 & & 77 & 77 & 77 & & 100 & 100 & 100 \\
\hline 21 & 25 & & 25 & 25 & 25 & & 25 & 27 & 27 & & 27 & 27 & 27 & & 4 & 4 & 4 \\
\hline 22 & 2 & & 2 & 2 & 2 & & 2 & & & & & & & & & & \\
\hline 23 & 52 & & 52 & 52 & 52 & & 52 & 52 & 52 & & 89 & 52 & 52 & & 89 & 52 & 52 \\
\hline 24 & 0 & & 0 & 0 & 0 & & 0 & & & & & & & & & & \\
\hline 25 & 27 & & 27 & 27 & 27 & & 27 & 37 & 37 & & & 37 & 37 & & 0 & 37 & 37 \\
\hline
\end{tabular}

$K_{t}=K, R_{t}=R, Q_{t}=Q, \omega_{i}=\omega$ and $c_{t}=c$. Since no shortages are allowed, we ignored the unit production cost (i.e., $c=0$ ). The rest of the parameters of the experimental set were: $K \in\{75,50,25\}, R \in\{154,152, \ldots, 54,52\}$, $M \in\{1,1.75,2.5,3.5,4.5,5.5\}$. For warming costs, we used $\omega \in\{0,0.05,0.1,0.15, \ldots, 0.85,0.9,0.95\}$ and $\omega>1$ as a special case $(Q=R)$. Note that, since $h=1$, one can interpret the values of $\omega$ as the ratio of unit warming cost to unit holding cost per period. As discussed above, the minimum cost is achieved when the warm process threshold is set at the point of indifference; therefore, in our numerical study, we used $Q=\hat{Q}$, unless stated explicitly otherwise.

All instances were solved on an IBM Pentium III using the forward DP algorithm provided in the Appendix with a complexity $O\left(N^{3}\right)$ after smoothing the given individual demands to ensure feasibility, which is done in $O(N)$ time.

\subsubsection{Sensitivity}

As a representative sample of our results, consider the medium-demand case $(M=2.5)$ tabulated in Table 1 ; for brevity only the first 25 periods of the optimal solution are presented. In this table, periods in which there is no production and no warming are left blank, italics indicate periods in which the process is kept warm at the end of the previous period.

We notice that for some values of $\omega$, the optimal solution is a lot-for-lot policy; this is actually the case for all parameter combinations for which the point of indifference $\hat{Q}$ is found to be less than or equal to zero $(\Rightarrow Q=0)$. This is to be expected since keeping the process warm until the next period is more beneficial than incurring the cost of a cold setup in the next period even if there is no production done in the current period (indicated by a 0 ). Incidentally, in such cases, the process is always kept warm. For positive $\hat{Q}$, batching occurs as expected. As $\omega$ increases, batching becomes more beneficial and run sizes increase.

The impact of the cold setup cost, $K$, is similar to that of $\omega$ in inducing batching albeit in the opposite direction, and it is more pronounced. As $K$ decreases, the point of indifference $\hat{Q}$ increases; thus, the option of keeping the process warm loses its appeal since it would imply excessively large run sizes resulting in higher carrying costs. Hence, for small $K$, the optimal production schedule is closer to a lot-for-lot policy with a few big batches inserted. Hence, when $K$ decreases, the number of periods in which there is production increases; however, there are more cold setups done than warm setups. 
For the unreported cases of low and high demands, we observed less sensitivity of the optimal schedule to the system parameters. We also observed that the impact of capacity $R$ is primarily through $\hat{Q}$ except for cases with very-tight capacity levels.

Our results indicate that the warm process threshold, $\hat{Q}$, plays a more critical role in warm/cold process decisions than the individual values of system parameters. This observation motivated us to investigate the special case of the "warm-only-if-at-capacity" policy, where $Q_{t}=R_{t}$ for all $t$. This policy would give the optimal solution when $\omega_{t} \geq h_{t}$ since $\hat{Q}_{t} \leq 0$ for all $t$. In other instances, it is a heuristic corresponding to a constrained solution of the problem. This policy is important because it also corresponds to the cases where undertime options are deliberately not used by management even though they are available. It may also be viewed as a "big-bucket version" of the DLSP. The best schedule obtained under the warm-only-if-at-capacity policy for our base problem is also given in Table 1. Since it corresponds to the optimal solution when $\omega>1$, we see most batching in this case. Furthermore, the imposed policy encourages batching in the best solution for large $K$ values; but, for small $K$, it gives a schedule similar to that obtained for moderate unit warming costs. This tendency was also validated for low and high-demand scenarios with other values of the cost parameters.

Similarly, we observe that the deviation of the total cost from the optimal value under the $Q=R$ policy decreases as $\omega$ increases, for all demand levels and setup cost values. (For an instance of $R$, we refer the reader to Fig. 1). For smaller demand levels and smaller cold setup costs, the de- viation becomes zero at smaller values of $\omega$. The speed of convergence is more sensitive to the changes in $K$.

\subsubsection{Managerial implications: capacity selection}

Next, we study capacity issues for warm/cold processes. We report our findings on the medium-demand case with $K=75$ for a broad range of capacity values, $R=[52,154]$, where $R=52$ corresponds to the minimum capacity level for which a feasible solution exists under the given demand pattern. Throughout, we assume that $Q=\hat{Q}$, which provides the lowest attainable cost. (Note that as $R$ changes, so does $\hat{Q}$.) We focus on the behavior of the total-cost and its components as the capacity of the process changes and report them in Fig. 1-4. The reported costs are for the entire problem horizon $(N=100)$.

We observe a non-monotonic behavior in the total cost with respect to capacity. As capacity decreases, the total cost initially decreases; then, there is an increase for all values of $\omega$. For large values of $\omega$ (and for the imposed warm-onlyif-at-capacity policy), the total-cost curve fluctuates and, in some instances, exhibits sudden jumps (see Fig. 2). This erratic behavior is best explained through individual cost components.

First consider the setup costs depicted in Fig. 4. As $R$ decreases, the incurred setup cost decreases almost monotonically followed by a sudden downward jump to the value of $K=75$ after which it remains flat. That the setup cost equals a value of $K$ implies that there is a single cold setup over the entire horizon of the problem in the optimal solution. This instance corresponds to the capacity level at which $\hat{Q}$ ceases to attain a positive value as $R$ decreases; hence, the process can be kept warm throughout the horizon

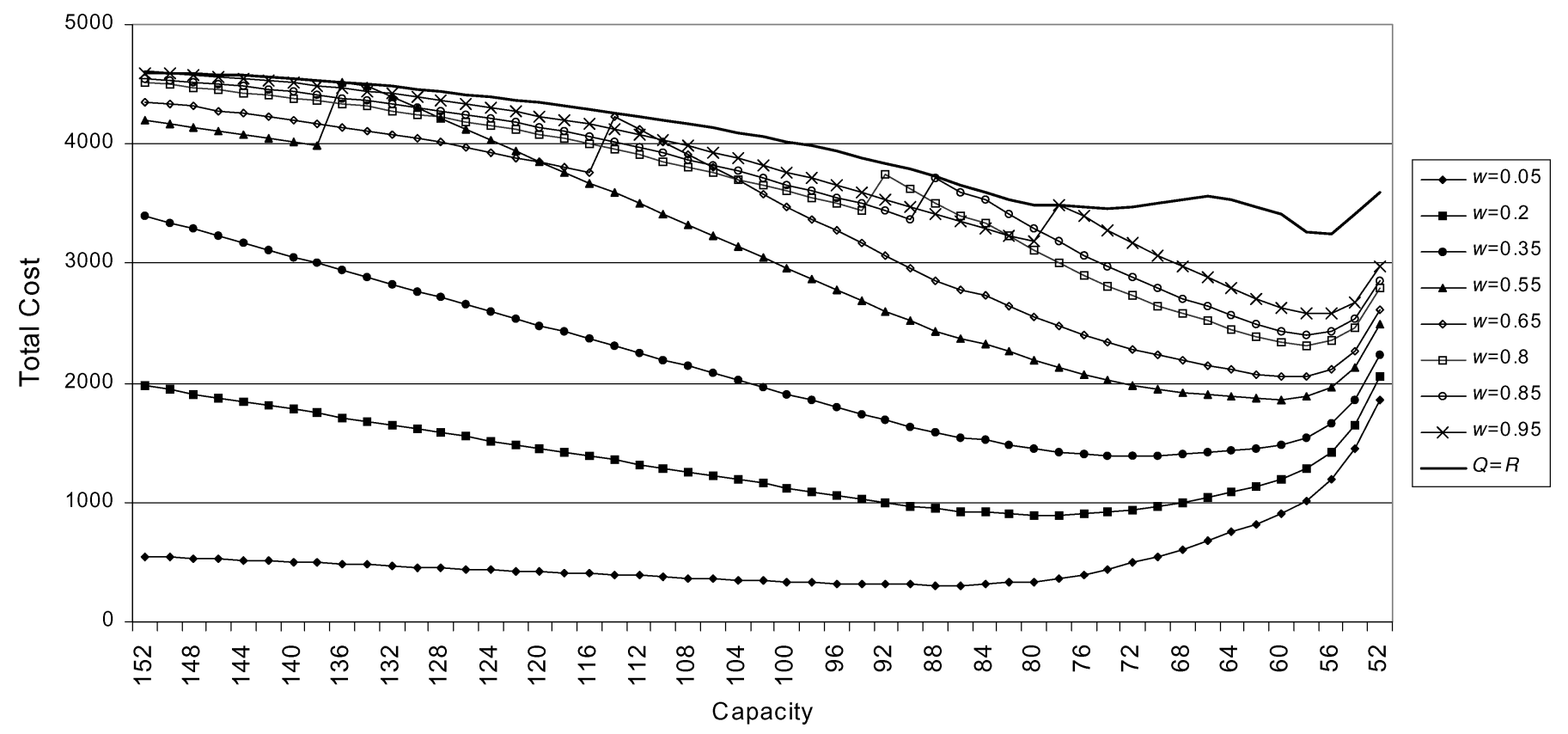

Fig. 1. Total cost against capacity (medium demand, $K=75$ ). 


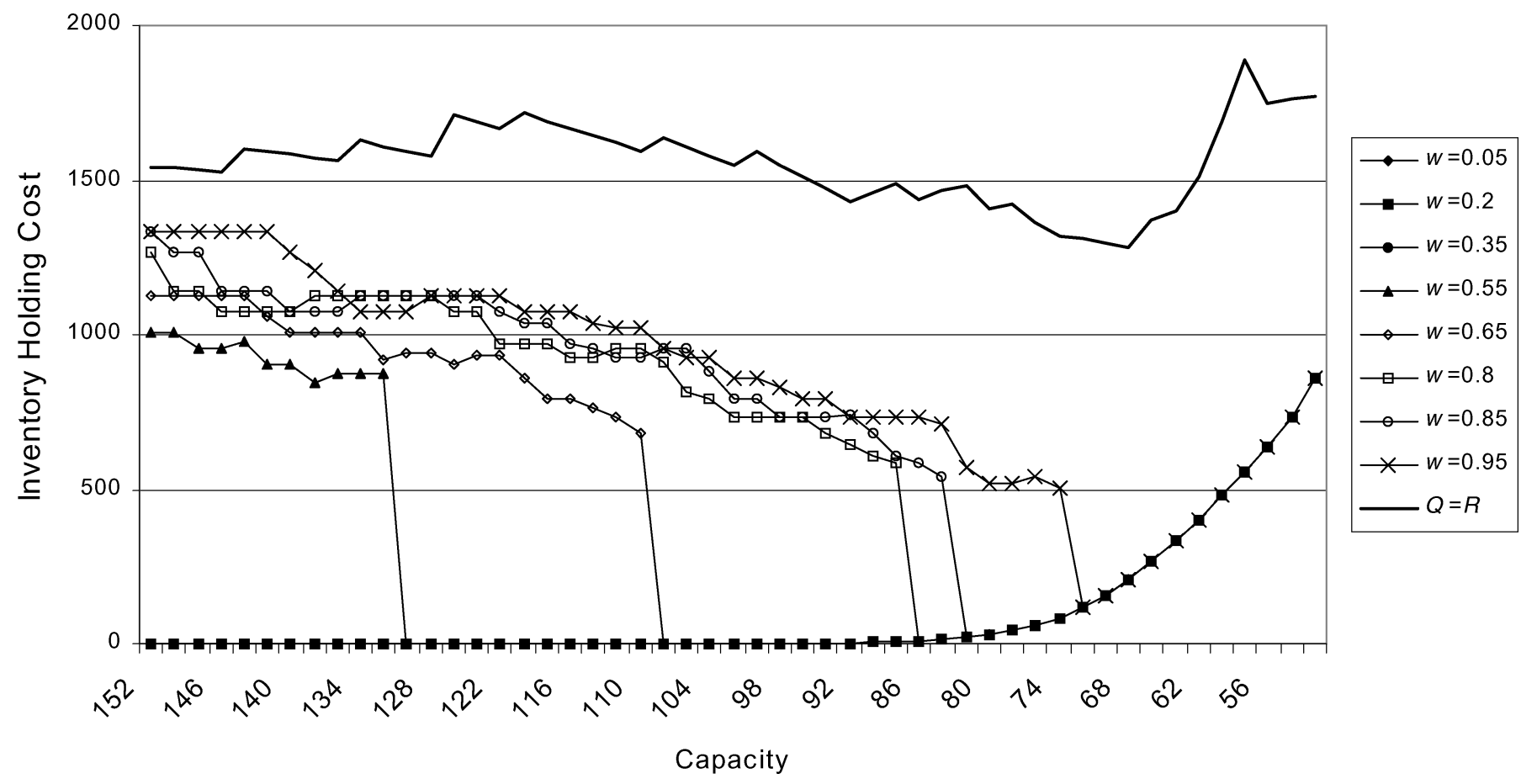

Fig. 2. Inventory holding cost against capacity (medium demand, $K=75$ ).

even if no production is done. Note that this happens at higher capacity levels for smaller $\omega$.

For the warming cost depicted in Fig. 3, we observe an opposite behavior. As $R$ decreases, the warming cost increases albeit non-monotonically until a sudden upward jump, followed by an almost steady decrease. The jump coincides with the same capacity level observed in the behavior of setup costs. Similarly, the jump in warming costs occurs at higher capacity levels for smaller $\omega$. The behavior of the two cost components vis a vis each other illustrates the

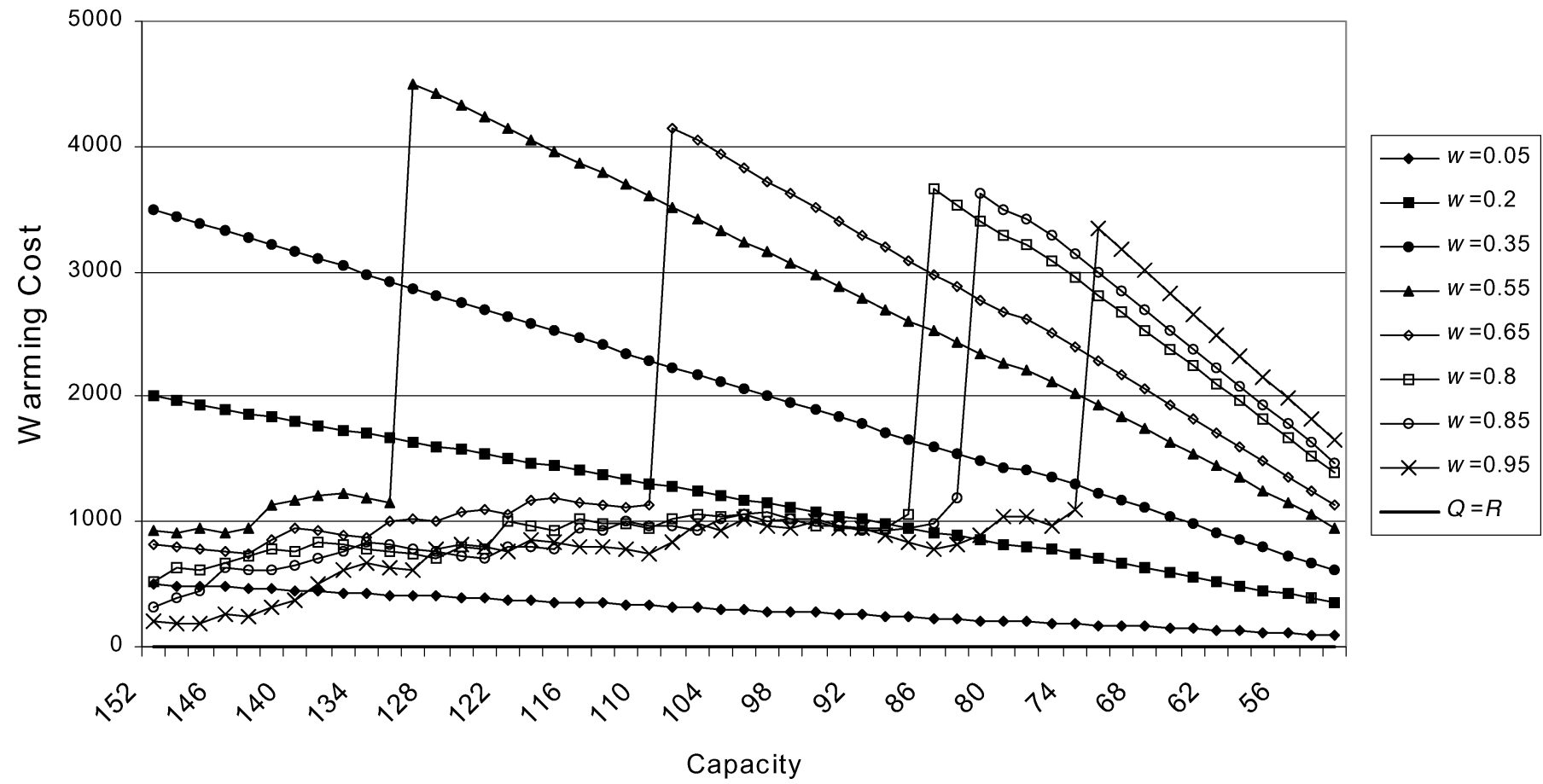

Fig. 3. Warming cost against capacity (medium demand, $K=75$ ). 


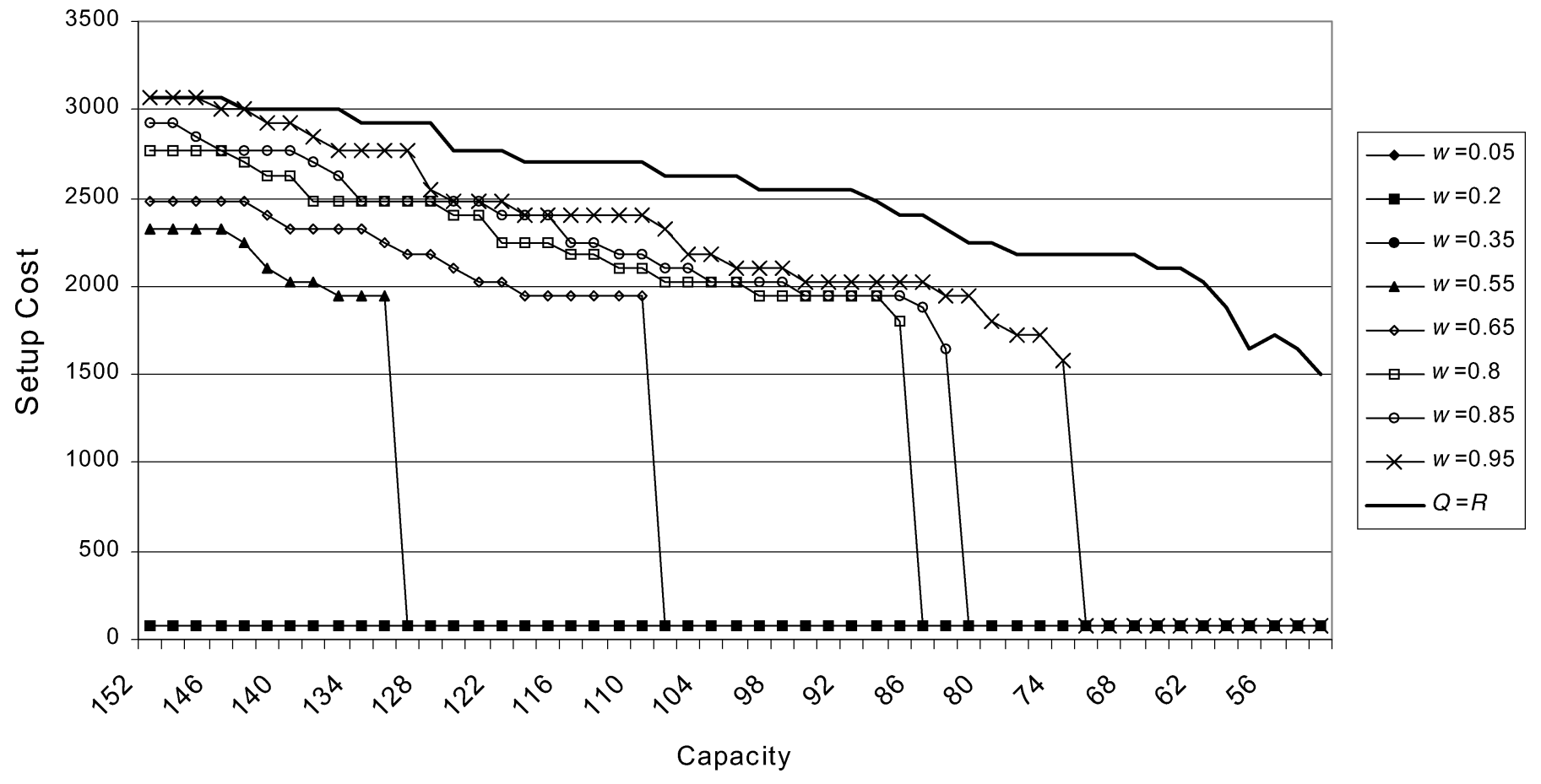

Fig. 4. Setup cost against capacity (medium demand, $K=75$ ).

fundamental trade-off in the presence of warm/cold processes. In fact, a closer examination of the numerical results reveals that the two cost components go in tandem. This is intuitive but still important to observe.

The main component that causes the total-cost curve to exhibit a bumpy behavior is the inventory holding cost depicted in Fig. 2. As $R$ decreases, the holding cost tends to decrease, as expected, since with lower $R$ values, less inventory is carried. At the capacity level where $\hat{Q} \leq 0$, the production schedule is the lot-for-lot policy; hence, no inventory is carried in those cases and we observe zero holding costs after this point, as $R$ decreases. However, if $R$ decreases further, we begin to see the effects of prior demand smoothing to ensure feasibility. That is, to ensure feasibility, the solution is forced a priori to carry more and more inventory in advance as the capacity tightness increases. This increasing portion of the inventory cost is what causes the increase in the total cost as $R$ gets smaller.

Although non-monotonic, we observe that there is an overall "convex" trend in the total cost with respect to the capacity limit. That is, there is an "optimal" capacity level which minimizes the total costs over the horizon. The optimal capacity level appears to increase as $\omega$ decreases. The analysis of the total cost provides further managerial implications regarding capacity selection and use of undertime options. Next, we discuss such issues.

The model and the solution procedures discussed herein provide a manager with the tools to determine the optimal capacity level in the presence of warm/cold processes, as well. For example, from Fig. 1, it is easy to see that an economically rational manager would choose $R=72$ as the optimal capacity level when $\omega=0.35$ for the numerical setting considered. (However, we should point out that this conclusion is based on a single known sample path of demands and cannot be generalized to a more realistic scenario of stochastic demands. For brevity, in our discussion herein, we consider such robustness issues to be outside the scope of our analysis, which can be addressed in a simulation context.) Yet, the question remains: what are the implications of suboptimal capacity decisions? In particular, what happens if the manager ignores the availability of the undertime option?

We consider two such scenarios. In the first case, the manager restricts warm process decisions solely to instances in which the production quantity in a period is equal to the capacity limit; that is, the manager sets $Q=R$ and chooses the best capacity level accordingly. Note that the manager is aware of the advantages of keeping a process warm but behaves as if $\omega$ is prohibitively high $(\Rightarrow \hat{Q} \geq R)$. In the second case, the manager totally ignores the possibility of keeping the process warm and bases the capacity selection decision on the solution of the classical (uncapacitated) problem. Specifically, in this case, the best capacity is selected to equal the maximum production quantity obtained in the WagnerWhitin solution. In Table 2, we present a representative sample of our findings where $R_{\mathrm{opt}}^{*}, R_{1}^{*}$ and $R_{2}^{*}$ are, respectively, the optimal and the best capacity levels selected for the first and second cases. We let $\Delta_{i} \%$ (for $i=1,2$ ) denote the respective percentage deviations in total costs with respect to the total cost under the optimal capacity decision, 
Table 2. Impact of capacity selection policies on total costs (medium demand, $K=75$ )

\begin{tabular}{rcccccccrrrrr}
\hline & \multicolumn{10}{c}{$\omega$} \\
\cline { 2 - 10 } & 0 & 0.05 & 0.15 & 0.25 & 0.35 & 0.45 & 0.55 & 0.65 & 0.75 & 0.85 & 0.95 \\
\hline$\Delta_{1}$ & 1389.6 & 287.5 & 90 & 40.8 & 19.5 & 9.3 & 5.6 & 3.3 & 2 & 1.1 & 0.3 \\
$\Delta_{2}$ & 0 & 82.8 & 116.8 & 135.5 & 151.4 & 169.2 & 127 & 113.5 & 101.5 & 90.1 & 78.5 \\
\hline
\end{tabular}

and compute it as follows:

$$
\Delta_{i} \%=\frac{\left(T C_{R=R_{i}^{*}}-T C_{R=R_{\mathrm{opt}}^{*}}\right)}{T C_{R=R_{\mathrm{opt}}^{*}}} \times 100
$$

We find that $R_{1}^{*}<R_{\mathrm{opt}}^{*}<R_{2}^{*}$ for all $\omega$. This implies that ignoring potential benefits of warm processes results in selecting a capacity level higher than the optimal schedule necessitates, yielding a lower equipment utilization rate and possibly lower rates of Return On Investment (ROI). On the other hand, imposing the warm-only-if-at-capacity policy results in selecting a capacity level lower than the optimal. Thus, it yields a higher equipment utilization rate and possibly higher ROI. This apparent efficiency may be the reason behind the popularity of this policy among practitioners. However, the ensuing tightness of capacity, in fact, increases the total operating costs incurred. Operating with $R_{1}^{*}$ results in an excessively large cost differential for low unit warming costs; as $\omega$ increases, the differential vanishes in the limit, as expected. The cost differential monotonically decreases over $\omega$. When $R_{2}^{*}$ is used instead of $R_{\text {opt }}^{*}$, interestingly, the cost differential exhibits a concave behavior over $\omega$. It increases very steeply for low $\omega$, is concave over a large range of unit warming cost, and decreases slowly for large $\omega$. Thus, total ignorance of the undertime option results in the worst performance (more than $100 \%$ deviation from the optimal) over a broad range of parameter values. Its concave behavior also implies that management would most benefit from the use of the undertime option in capacity selection decisions for moderate values of the unit warming cost.

In conclusion, the presence of warm/cold processes impacts total operating costs not only by yielding differently structured production schedules compared to the classical settings, but also through optimal capacity selection decisions taking into account the undertime option.

\section{Conclusions and future work}

In this work, we have considered lot sizing decisions for a process which can be kept warm for the next period at an additional linear cost if the production quantity in the current period is at least a positive threshold amount. We have established the structure of the optimal production schedule and the conditions under which a forward polynomial-time solution is possible. As a special case, we also presented a linear time solution. Through a numerical study, we have also investigated the impact of a warm process option on the required capacity for a given stream. Although our focus has been to obtain the optimal solution, the related issue of heuristic solutions remains an open research area.

We can conjecture that forward heuristics, in general, would perform relatively better for the case where $k_{t}=0$, since, in this case, a forward optimal solution is possible. In an unreported simulation study, we examined the performance of two forward heuristics (suggested by one of the anonymous referees) based on the principle of spreading over periods via the warm process threshold of the unconstrained Wagner-Whitin solution. In both heuristics, the warm process option is ignored. In one of the heuristics, the Wagner-Whitin solution is obtained by using the warm setup cost value as the setup cost throughout the horizon. In the other, the heuristic solution is obtained by assuming the setup cost as the cold setup cost originally given in the problem. As such, both heuristics are simple heuristics and performed very poorly. However, more sophisticated heuristics may be developed which generate solutions in a forward manner using the structure of optimal schedules (Corollary 3) and a stopping rule in the same spirit of the heuristics available for the (un)capacitated dynamic lot sizing problem. Development of such approximate solutions for a warm/cold process constitutes an interesting research area. In addition, metaheuristics such as tabu search and simulated annealing are also interesting venues of research.

Furthermore, most real-world problems exhibit demand uncertainty Hence, the robustness of the solutions to such changes in demands is also important. Our structural results indicate that the option of keeping the process warm enables longer production series vis a vis the CLSP setting. One can conjecture that a warm/cold process would be less susceptible to the "nervousness" phenomenon, since production is kept going over a number successive periods. However, it is not possible to say a priori which type of heuristic would be the best and it is another fertile research topic.

We intend to visit these issues in our future work.

\section{References}

Aggarwal, A. and Park, J.K. (1989) Sequential searching in multidimensional monotone arrays. Research Report RC 15128, IBM T.J. Watson Research Center, Yorktown Heights, NY.

Aggarwal, A. and Park, J.K. (1993) Improved algorithms for economic lot size problems. Operations Research, 41(3), 549-571. 
Agra, A. and Constantino, M. (1999) Lot sizing with backordering and start-ups: the case of Wagner-Whitin costs. Operations Research Letters, 25, 81-88.

Allahverdi, A., Gupta, J.N.D. and Aldowaisan, T. (1999) A review of scheduling research involving setup considerations. Omega, 27, 219239.

Baker, K.R., Dixon, P., Magazine, J. and Silver, E.A. (1978) An algorithm for the dynamic lot-size problem with time-varying production capacity constraints. Management Science, 24(16), 1710-1720.

Bitran, G.R. and Matsuo, H. (1986) Approximation formulations for the single-product capacitated lot size problem. Operations Research, 34(1), 63-74.

Bitran, G.R. and Yanasse, H.H. (1982) Computational complexity of the capacitated lot size problem. Management Science, 28(10), 1174 1186.

Blackburn, J.D. and Kunreuther, H. (1974) Planning horizons for the dynamic lot size model with backlogging. Management Science, 21(3), 251-255.

Bruggemann, W. and Jahnke, H. (2000) The discrete lot-sizing and scheduling problem: complexity and modification for batch availability. European Journal of Operational Research, 124, 511528.

Eiamkanchanalai, S. and Banerjee, A. (1999) Production lot sizing with variable production rate and explicit idle capacity cost. International Journal of Production Economics, 59, 251-259.

Eppen, G,D. and Martin, R.K. (1987) Solving multi-item capacitated lot-sizing problems using variable redefinition. Operations Research, 35(6), 832-848.

Eppstein, D. (1990) Sequence comparison with mixed convex and concave costs. Journal of Algorithms, 11, 85-101.

Fleischmann, B. (1990) The discrete lot-sizing and scheduling problem. European Journal of Operational Research, 44, 337-348.

Florian, M. and Klein, M. (1971) Deterministic production planning with concave costs and capacity constraints. Management Science, 18(1), $12-20$.

Gallego, G. (1993) Reduced production rates in the economic lot scheduling problem. International Journal of Production Research, 31, 10351046.

Hindi, K.S. (1995a) Efficient solution of the single-item, capacitated lotsizing problem with start-up and reservation costs. Journal of the Operational Research Society, 46(10), 1223-1236.

Hindi, K.S. (1995b) Solving the single-item, capacitated dynamic lot-sizing problem with startup and reservation costs by tabu search. Computers \& Industrial Engineering, 28(4), 701707.

Jagannathan, R. and Rao, M.R. (1973) A class of deterministic production planning problems. Management Science, 19(11), 1295 1300 .

Karimi, B. (2003) The capacitated lot sizing problem: a review of models and algorithms. Omega, 31(5), 365-378.

Karmarkar, U.S., Kekre, S. and Kekre, S. (1987) The dynamic lot-sizing problem with startup and reservation costs. Operations Research, 35(3), 389-398.

Loparic, M., Marchand, H. and Wolsey, L.A. (2003) Dynamic knapsack sets and capacitated lot-sizing. Mathematical Programming Series $B, 95,53-59$.

Love, S.F. (1973) Bounded production and inventory models with piecewise concave costs. Management Science, 20(3), 313318.

Manne, A.S. (1958) Programming of economic lot sizes. Management Science, 4(2), 115-135.

Moon, I., Gallego, G. and Simchi-Levi, D. (1991) Controllable production rates in a family production context. International Journal of Production Research, 29, 2459-2470.

Pochet, Y. and Wolsey L.A. (1995) Algorithms and reformulations for lot sizing problems. DIMACS Series in Discrete Mathematics and Theoretical Computer Science, 20, 245-293.
Robinson, E.P. and Sahin, F. (2001) Economic production lot sizing with periodic costs and overtime. Decision Sciences Journal, 32(3), 423452.

Silver, E.A. (1990) Deliberately slowing down output in a family production context. International Journal of Production Research, 28 , $17-27$.

Wagner, H.M. and Whitin T.M. (1958) Dynamic version of the economic lot size model. Management Science, 5(1), 89-96.

Wolsey, L.A. (1995) Progress with single-item lot-sizing. European Journal of Operational Research, 86, 395-401.

Zangwill, W.I. (1966) A deterministic multi-period production scheduling model with backlogging. Management Science, 13(1), 105119.

\section{Appendices}

\section{Appendix A: An illustrative example}

We consider the following example setting: $c_{t}=c, h_{t}=$ $h, \omega_{t}=\omega, K_{t}=K, k_{t}=k, R_{t}=R$ and $Q_{t}=Q$ for $t=$ $1, \cdots, N$. We set $N=25, c=0, h=1, \omega=0.85, K=15$, $k=0, R=10$ and $Q=7$. The demand over the problem horizon is given by $\mathbf{D}=\{4,2,4,4,3,7,9,1,6,4,10,2,1$, $5,8,2,9,2,5,2,7,3,4,5,8\}$.

Below, we present every step of a forward solution. At every step, we consider a $T$-period problem (i.e., a problem with the horizon length of $T$ starting from the very first period), and generate a set of possible production schedules of this $T$-period problem by imposing the condition $y_{t-1}=0$ for a period $t(1 \leq t \leq T)$. In Fig. A1, each row corresponds to such a schedule. We compute the cost of each suggested schedule such that the cost of the periods 1 through $t-1$ is the optimal cost obtained from the $(t-1)$-period problem, and the cost of the periods $t$ through $T$ computed afresh. The notation $\left(^{*}\right)$ denotes the schedule that yields the lowest cost as the optimal schedule for the $T$-period problem.

Note that, since $k=0$, we only need to consider the production sequences that consist of a single production series. Further note that, we directly use Theorem 4 in constructing the alternative schedules that need to be considered in an optimal solution.

Note that in this illustrative example, in period 7, the partition condition in Theorem 5 part (ii) is satisfied; hence, a partition occurs in period 7 . The production schedule obtained up to period 6 in the seven-period problem remains the same for any problem with a longer horizon length. In this example, in the optimal solution, there are eight production series. The solution is as follows: $\mathbf{X}=\{[6,0]$; $[7,4,0] ;[7,9,7,4,0] ;[10,3,0] ;[7,8,0] ;[9,9,0,0] ;[7,7,0]$; $[7,6]\}$. The optimal solution to the full problem is also depicted in Fig. A2 along with the demands indicated by a diamond mark.

\section{Appendix B: Forward solution DP algorithm}

We retain the labels for the corresponding system parameters in the model but switch to a vector notation, such 


\begin{tabular}{|c|c|c|c|c|}
\hline \multicolumn{5}{|c|}{ One-period problem: } \\
\hline$f_{0,0}^{*}=$ & $=0$ & $1=15$ & $f_{1,1}=15$ & $f_{1,1}^{*}=15$ \\
\hline \multicolumn{5}{|c|}{ Two-period problem: } \\
\hline$\{6,0\}$ & $f_{0,0}^{*}=0$ & $f_{1,2}=17$ & $f_{1,2}=17$ & $f_{1,2}^{*}=17$ \\
\hline$\Psi_{(1,1)},\{2\}$ & $f_{1,1}^{*}=15$ & $f_{2,2}=15$ & $f_{1,2}=30$ & \\
\hline \multicolumn{5}{|c|}{ Three-period problem: } \\
\hline$\{\mathbf{7}, \mathbf{3}, \mathbf{0}\}$ & $f_{0,0}^{*}=0$ & $f_{1,3}=24.55$ & $1,3=24.55$ & $f_{1,3}^{*}=24.55$ \\
\hline$\Psi_{(1,1)},\{6,0\}$ & $f_{1,1}^{*}=15$ & $f_{2,3}=19$ & $1,3=34$ & \\
\hline$\Psi_{(1,2)},\{4\}$ & $f_{1,2}^{*}=17$ & $f_{3,3}=15$ & $1,3=32$ & \\
\hline \multicolumn{5}{|c|}{ Four-period problem: } \\
\hline$\{\mathbf{7}, \mathbf{7}, \mathbf{0}, \mathbf{0}\}$ & $f_{0,0}^{*}=0$ & $f_{1,4}=32.55 \quad f$ & $f_{1,4}=32.55$ & $f_{1,4}^{*}=32.55$ \\
\hline$\Psi_{(1,1)},\{7,3,0\} \quad f_{1,1}^{*}$ & $f_{1,1}^{*}=15$ & $f_{2,4}=26.55$ & $f_{1,4}=41.55$ & \\
\hline$\Psi_{(1,2)},\{7,1\}$ & $f_{1,2}^{*}=17$ & $f_{3,4}=20.55$ & $f_{1,4}=37.55$ & \\
\hline$\Psi_{(1,3)},\{4\}$ & $f_{1,3}^{*}=24.55$ & $f_{4,4}=15$ & $f_{1,4}=39.55$ & \\
\hline \multicolumn{5}{|c|}{ Five-period problem: } \\
\hline$\{7,7,3,0,0\}$ & $f_{0,0}^{*}=0$ & $f_{1,5}=41.10$ & $f_{1,5}=41.10$ & \\
\hline$\Psi_{(1,1)},\{7,6,0,0\} \quad f_{1}^{\prime}$ & $f_{1,1}^{*}=15$ & $f_{2,5}=32.55$ & $f_{1,5}=47.55$ & \\
\hline$\Psi_{(1,2)},\{\mathbf{7}, \mathbf{4}, \mathbf{0}\}$ & $f_{1,2}^{*}=17$ & $f_{3,5}=23.55$ & $f_{1,5}=40.55$ & $f_{1,5}^{*}=40.55$ \\
\hline$\Psi_{(1,3)},\{7,0\}$ & $f_{1,3}^{*}=24.55 \quad f$ & $f_{4,5}=18$ & $f_{1,5}=42.55$ & \\
\hline$\Psi_{(1,4)},\{3\}$ & $f_{1,4}^{*}=32.55 \quad f$ & $f_{5,5}=15$ & $f_{1,5}=47.55$ & \\
\hline Six-period proble & em: & & & \\
\hline$\{7,7,7,3,0,0\}$ & $f_{0,0}^{*}=0$ & $f_{1,6}=61.65$ & \begin{tabular}{l|l|}
5 & $f_{1,6}=61.65$ \\
\end{tabular} & \\
\hline$\Psi_{(1,1)},\{7,7,6,0,0\}$ & $f_{1,1}^{*}=15$ & $f_{2,6}=50.10$ & $f_{1,6}=65.10$ & \\
\hline$\Psi_{(1,2)},\{7,7,4,0\}$ & $f_{1,2}^{*}=17$ & $f_{3,6}=36.10$ & $f_{1,6}=53.10$ & \\
\hline$\Psi_{(1,3)},\{\mathbf{7}, \mathbf{7}, \mathbf{0}\}$ & $f_{1,3}^{*}=24.55$ & $\begin{array}{l}f_{4,6}=27.55 \\
\end{array}$ & $5 f_{1,6}=52.10$ & $f_{1,6}^{*}=52.10$ \\
\hline$\Psi_{(1,4)},\{7,1\}$ & $f_{1,4}^{*}=32.55$ & $f_{5,6}=21.55$ & $5 f_{1,6}=54.10$ & \\
\hline$\Psi_{(1,5)},\{5\}$ & $f_{1,5}^{*}=41.10$ & $f_{6,6}=15$ & $f_{1,6}=56.10$ & \\
\hline Seven-period prob & blem: & & & \\
\hline$\{7,7,7,7,5,0,0\}$ & $f_{0,0}^{*}=0$ & $f_{1,7}=86.20$ & $f_{1,7}=86.20$ & \\
\hline$\Psi_{(1,1)},\{7,7,7,7,1,0\}$ & $f_{1,1}^{*}=15$ & $f_{2,7}=73.20$ & $f_{1,7}=88.20$ & \\
\hline$\Psi_{(1,2)},\{7,7,7,6,0\}$ & $f_{1,2}^{*}=17$ & $f_{3,7}=56.65$ & $5 f_{1,7}=67.65$ & \\
\hline$\Psi_{(1,3)},\{7,7,7,2\}$ & $f_{1,3}^{*}=24.55$ & \begin{tabular}{l|l}
5 & $f_{4,7}=39.65$ \\
\end{tabular} & $5 f_{1,7}=64.20$ & \\
\hline$\Psi_{(1,4)},\{7,7,5\}$ & $f_{1,4}^{*}=32.55$ & \begin{tabular}{l|l|}
5 & $f_{5,7}=28.10$ \\
\end{tabular} & $f_{1,7}=60.65$ & \\
\hline$\Psi_{(1,5)},\{\mathbf{7}, \mathbf{9}\}$ & $f_{1,5}^{*}=40.55$ & \begin{tabular}{l|l|}
5 & $f_{6,7}=17.55$ \\
\end{tabular} & $5 f_{1,7}=58.10$ & $f_{1,7}^{*}=58.10$ \\
\hline$\Psi_{(1,6)},\{9\}$ & $f_{1,6}^{*}=52.10$ & $0 f_{7,7}=15$ & $f_{1,7}=67.10$ & \\
\hline
\end{tabular}

as $\mathbf{D}[\cdot]$, etc. Furthermore, we introduce the following new notation: $\mathbf{s}[\cdot]$ is the setup cost actually incurred, $\mathbf{Z}[i][j]$ is defined as $f_{1}^{j-1}(1,0)+f_{1}^{i}(1,0)$ and is an intermediate variable, $\mathbf{Z}[i][$ best $[i]]$ is defined as $f_{1}^{i}(1,0)$ and denotes the cost of an optimal production policy for the $i$-period problem, and $\pi[i][j][t]$ is the production quantity in period $t$ when a cold setup is forced in period $j$ for an $i$-period problem. The algorithm is as follows:

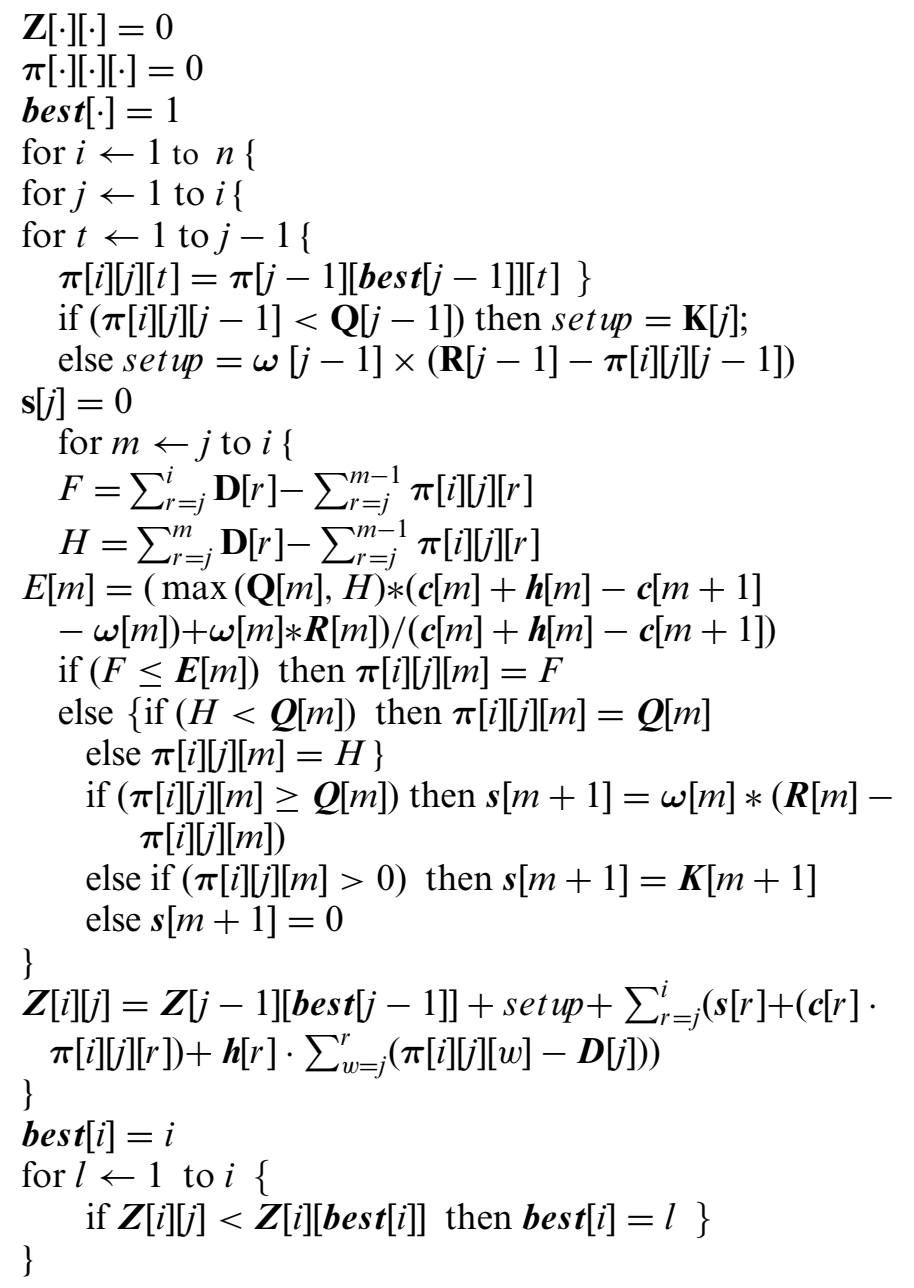

\section{Appendix C: Proofs}

Proof of Proposition 1. The proof is similar to that in Bitran and Yanasse (1982) and consists in defining a new inventory variable $I_{t-1}=y_{t-1}-\max _{\tau=0, \ldots, N-t}\left\{0, \sum_{j=t}^{t+\tau}\left(D_{j}-\right.\right.$ $\left.R_{j}\right)$ \} and rewriting the objective function in terms of the new inventory variable. The new objective function differs from the original one only by a constant, hence the result.

Proof of Theorem 1. Suppose the contrary (i.e., $z_{t} \times$ $\left.x_{t} \times\left[y_{t-1}-D_{t}\right]^{+}>0\right)$. That is, suppose a proposed production plan suggests $x_{t-1}<Q_{t-1},\left[y_{t-1}-D_{t}\right]^{+}>0$, and $x_{t}>0$. Since the production in period $t-1$ is less than the warm process threshold, the process will be cold in period $t$. As in the classical lot sizing problem,

Fig. A1. The production schedules. 


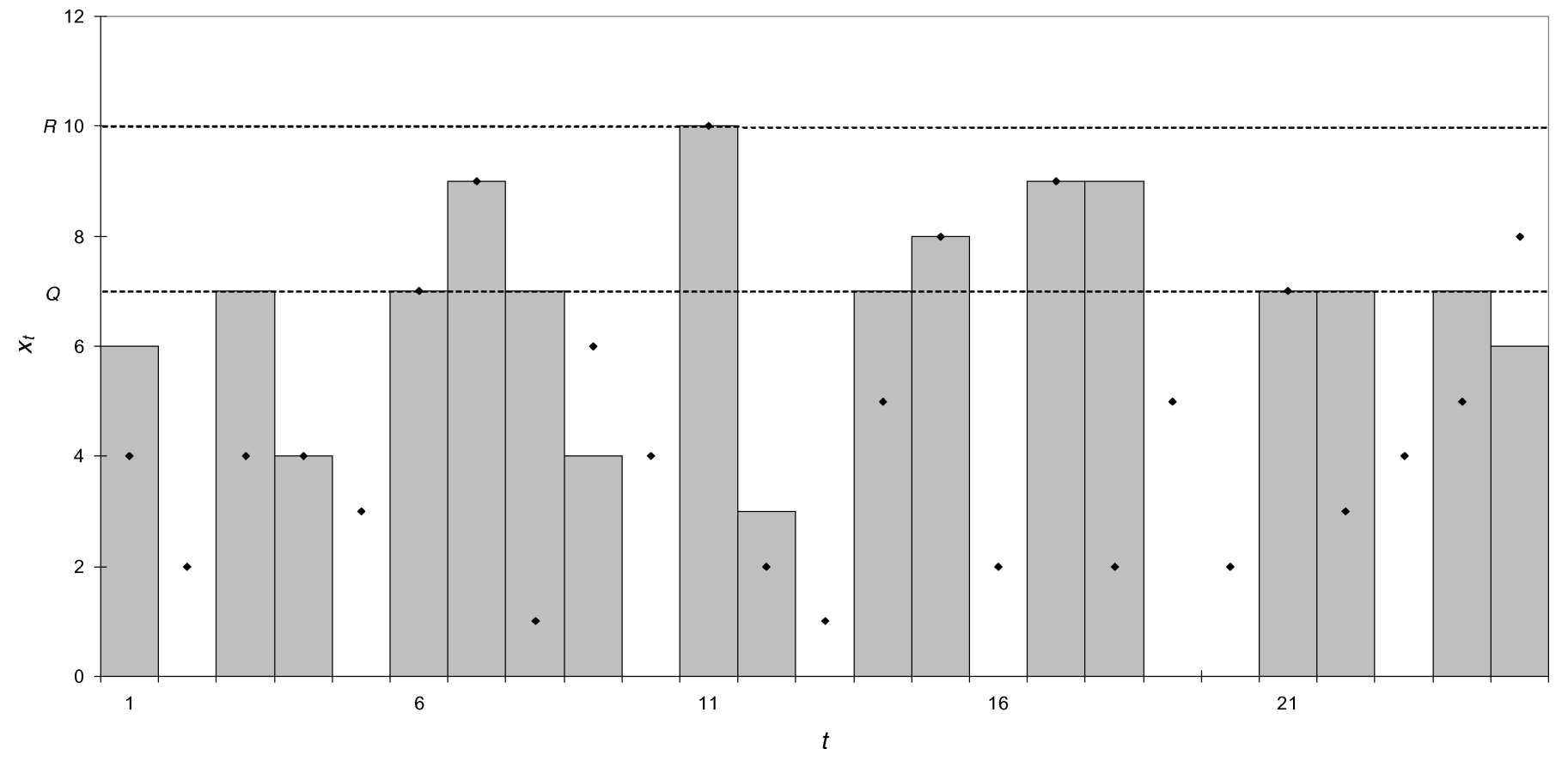

Fig. A2. Illustrative example: demand pattern and optimal production schedule.

the solution can be improved; hence, it cannot be optimal. Therefore, whenever $z_{t}=1$ production is done if and only if the starting inventory is strictly less than the demand for that period. With $z_{t}=0$, however, there is no such restriction on the production plan; hence, the optimal production plan satisfies the stated condition.

Proof of Proposition 2. With respect to problem (P), we only change the demands in periods $u$ and $v-1$ in $\left(\mathrm{P}^{\prime}\right)$. From Proposition 1, feasibility is ensured for problem $\left(\mathrm{P}^{\prime}\right)$. In both problems, under the same production schedule, the net demands in periods $u$ through $v-1$ are the same. Therefore, the costs are also the same. Hence, the result.

Proof of Lemma 1. (i) Consider the production series $\Psi_{u v}$ and let $m$ be the last period with production within this series. First, suppose $u \leq t=m=v-1$. In this case, $x_{t}=\left[D_{t}-y_{t-1}\right]^{+}$and, by definition, $E_{t}>\left[D_{t}-y_{t-1}\right]^{+}$; therefore, $x_{t} \ngtr E_{t}$. Hence, the result. Next, suppose $u \leq t \leq m<v-1$ or $u \leq t<m=v-1$. We will prove by contradiction. In particular, we will show that any production series that violates the above result can be improved and, hence cannot be optimal. Suppose that series $\Psi_{u v}$ is feasible but violates the above lemma, such that $R_{t} \geq x_{t}>E_{t}$ for some $t(u \leq t \leq m)$ where $t=\max \left\{i: x_{i}>E_{i}\right\}$. Consider another production series $\Psi_{u v}^{\prime}$ such that $\Psi_{u v}^{\prime}=$ $\left\{x_{u}^{\prime}=x_{u}, \ldots, x_{t-1}^{\prime}=x_{t-1}, x_{t}^{\prime}=E_{t}+e_{0}, x_{t+1}^{\prime}=x_{t+1}+e_{1}\right.$, $\left.x_{t+2}^{\prime}=x_{t+2}+e_{2}, \ldots, x_{v-1}^{\prime}=x_{v-1}+e_{v-1-t}\right\}$ where $x_{t}^{\prime}<$ $x_{t}, \quad 0 \leq e_{i}:=x_{t+i}^{\prime}-x_{t+i} \leq s_{i}:=\left[\min \left(R_{t+i}, E_{t+i}^{\prime}\right)-x_{t+i}\right]^{+}$ for $1 \leq i \leq v-1-t$ and $\sum_{i=1}^{v-1-t} e_{i}=\left(x_{t}-x_{t}^{\prime}\right)$. (Throughout in our proofs, all entities with notation (') retain their original definition and indicate recomputation for a new sequence.)

Note that in this new production series, for $t+1 \leq$ $i \leq v-1, y_{i}^{\prime}=y_{i}-\left(x_{t}-x_{t}^{\prime}\right)+\sum_{j=t+1}^{i}\left(x_{j}^{\prime}-x_{j}\right)$, hence, $y_{i}^{\prime}$ $\leq y_{i}$; therefore, $\left[D_{i}-y_{i-1}^{\prime}\right]^{+} \geq\left[D_{i}-y_{i-1}\right]^{+}$implying $E_{i}^{\prime} \geq$ $E_{i}$. Furthermore, due to the construction of the new series, $x_{i}^{\prime} \leq E_{i}^{\prime}$. Let $\Delta$ denote the cost difference between production series $\Psi_{u v}^{\prime}$ and $\Psi_{u v}$. Then, $\Delta=-\left(x_{t}-x_{t}^{\prime}\right)\left(c_{t}+h_{t}-\right.$ $\left.\omega_{t}\right)+\sum_{i=t+1}^{v-1} c_{i}\left(x_{i}^{\prime}-x_{i}\right)+\sum_{i=t+1}^{v-1} h_{i}\left(y_{i}^{\prime}-y_{i}\right)-\sum_{i=t+1}^{m} \omega_{i}$ $\left(x_{i}^{\prime}-x_{i}\right)+\sum_{i=m+1}^{v-1}\left(k_{i}+\omega_{i}\left(R_{i}-x_{i}^{\prime}\right) \delta_{i+1}^{\prime}\right) \delta_{i}^{\prime}$ where $\delta_{i}^{\prime}$ is a binary variable indicating whether or not production is done in period $i$. Noting that $\left(x_{t}-x_{t}^{\prime}\right)=\sum_{i=t+1}^{v-1}\left(x_{i}^{\prime}-x_{i}\right)$ and $y_{i}^{\prime}=y_{i}-\left(x_{t}-x_{t}^{\prime}\right)+\sum_{j=t+1}^{i}\left(x_{j}^{\prime}-x_{j}\right)$, and arranging the terms, we get $\Delta=-\sum_{i=t+1}^{v-1}\left[\left(x_{i}^{\prime}-x_{i}\right)\left(c_{t}+h_{t}-\omega_{t}+\right.\right.$ $\left.\left.\sum_{j=t+1}^{i-1} h_{j}\right)\right]-\sum_{i=t+1}^{v-1}\left(x_{i}^{\prime}-x_{i}\right) \sum_{j=i}^{v-1} h_{j}+\sum_{i=t+1}^{v-1} c_{i}\left(x_{i}^{\prime}-\right.$ $\left.x_{i}\right)-\sum_{i=t+1}^{m-1} \omega_{i}\left(x_{i}^{\prime}-x_{i}\right)-\omega_{m}\left(x_{m}^{\prime}-x_{m}\right) \delta_{m+1}^{\prime}+\sum_{i=t+1}^{v-1} h_{i}$ $\sum_{j=t+1}^{i}\left(x_{j}^{\prime}-x_{j}\right)+\sum_{i=m+1}^{v-1}\left(k_{i}+\omega_{i}\left(R_{i}-x_{i}^{\prime}\right) \delta_{i+1}^{\prime}\right) \delta_{i}^{\prime}$. Using the equivalence between $\sum_{i=t+1}^{v-1}\left(x_{i}^{\prime}-x_{i}\right) \sum_{j=i}^{v-1} h_{j}$ and $\sum_{i=t+1}^{v-1} h_{i} \sum_{j=t+1}^{i}\left(x_{j}^{\prime}-x_{j}\right)$, we finally obtain:

$$
\begin{aligned}
\Delta= & \sum_{i=t+1}^{v-1}\left[\left(x_{i}^{\prime}-x_{i}\right)\left(c_{i}-\left(c_{t}+h_{t}-\omega_{t}+\sum_{j=t+1}^{i-1} h_{j}\right)\right)\right] \\
& -\sum_{i=t+1}^{m} \omega_{i}\left(x_{i}^{\prime}-x_{i}\right) \delta_{i+1}^{\prime}+\sum_{i=m+1}^{v-1}\left(k_{i}+\omega_{i}\left(R_{i}-x_{i}^{\prime}\right) \delta_{i+1}^{\prime}\right) \delta_{i}^{\prime} .
\end{aligned}
$$

If $\sum_{i=1}^{m-t} s_{i} \geq x_{t}-E_{t}$, then one can construct a feasible series $\Psi_{u v}^{\prime}$ such that $m^{\prime}=m$ (where $m^{\prime}$ is defined as is $m$ for 
the series $\left.\Psi_{u v}^{\prime}\right), e_{i}=0$ for $m-t+1 \leq i \leq v-1-t$ and $\sum_{i=1}^{m-t} e_{i} \geq x_{t}-E_{t}$ with $e_{0} \leq 0$. Then, $\Delta=\sum_{i=t+1}^{m}\left[\left(x_{i}^{\prime}-\right.\right.$ $\left.\left.x_{i}\right)\left(c_{i}-\omega_{i} \delta_{i+1}^{\prime}-\left(c_{t}+h_{t}-\omega_{t}+\sum_{j=t+1}^{i-1} h_{j}\right)\right)\right]$. Since $c_{i}-$ $\omega_{i} \delta_{i+1}^{\prime}<\left(c_{t}+h_{t}+\sum_{j=t+1}^{i-1} h_{j}\right)$ due to the assumed marginal production cost structure and $\left(x_{i}^{\prime}-x_{i}\right) \geq 0$ for all $i \geq t+1$, we have $\Delta<0$. Thus, $\Psi_{u v}^{\prime}$ with $x_{t}^{\prime} \leq E_{t}$ yields a lower cost than $\Psi_{u v}$; therefore, $\Psi_{u v}$ cannot be optimal. If, however, $\sum_{i=1}^{m-t} s_{i}<x_{t}-E_{t}$, one can construct another $\Psi_{u v}^{\prime}$ such that $m^{\prime}=m+1, e_{0}=\max \left(Q_{t},\left[D_{t}-\right.\right.$ $\left.\left.y_{t-1}\right]^{+}\right)-E_{t}, \quad e_{i}=s_{i}$ for $1 \leq i \leq m-t$, and $e_{m+1-t}=$ $\left[\left(\left(k_{t+1}+R_{t} \omega_{t}-\max \left(Q_{t},\left[D_{t}-y_{t-1}\right]^{+}\right) \omega_{t}\right) /\left(c_{t}+h_{t}-c_{t+1}\right)\right)\right.$ $+q$ ] where $q=x_{t}-E_{t}-\sum_{i=1}^{m-t} e_{i}$, and $e_{i}=0$ for $m-t+2 \leq i \leq v-1-t$. Note that this series is feasible since $e_{m+1-t} \leq R_{m+1}$ due to non-decreasing capacities. (Also, note that we construct a series such that $e_{0} \leq 0$ in this case as well.) Then,

$$
\begin{aligned}
\Delta= & \sum_{i=t+1}^{m-1}\left[\left(x_{i}^{\prime}-x_{i}\right)\left(c_{i}-\omega_{i}-\left(c_{t}+h_{t}-\omega_{t}+\sum_{j=t+1}^{i-1} h_{j}\right)\right)\right] \\
& +\left(x_{m}^{\prime}-x_{m}\right)\left(c_{m}-\left(c_{t}+h_{t}-\omega_{t}+\sum_{j=t+1}^{m-1} h_{j}\right)\right) \\
& +k_{m+1}+\left(R_{m}-x_{m}^{\prime}\right) \omega_{m} \\
& +\left(\left[\frac{k_{t+1}+R_{t} \omega_{t}-\max \left(Q_{t},\left[D_{t}-y_{t-1}\right]^{+}\right) \omega_{t}}{c_{t}+h_{t}-c_{t+1}}+q\right]\right. \\
& \left.\times\left[c_{m+1}-c_{t}+\omega_{t}-\sum_{j=t}^{m} h_{j}\right]\right) .
\end{aligned}
$$

We need to consider the two possible values that $s_{m-t}^{\prime}$ may take on. First, suppose that $s_{m-t}^{\prime}=R_{m}-x_{m}^{\prime}$, which implies that $x_{m}^{\prime}=R_{m}$. Then, due to the assumed cost structure, $\Delta<0$. Next, suppose that $s_{m-t}^{\prime}=E_{m}^{\prime}-x_{m}^{\prime}$. Then, $x_{m}^{\prime}=$ $E_{m}^{\prime}$; using the defining expression for $E_{m}^{\prime}$, it is again easy to show that $\Delta<0$. Hence, $\Psi_{u v}^{\prime}$ yields a lower cost than $\Psi_{u v}$; therefore, $\Psi_{u v}$ cannot be optimal. If the newly constructed $\Psi_{u v}^{\prime}$ has $x_{m+1}^{\prime} \leq E_{m+1}^{\prime}$, we conclude our proof. Otherwise, we carry the same argumentation over periods $m+1$ and onward successively. Hence, the result.

(ii) Follows immediately from part (i).

Proof of Lemma 2. We will prove by contradiction. Suppose a feasible production series $\Psi_{u v}$ where for some $t, x_{t}=$ $R_{t}\left(>Q_{t}\right), 0<x_{t+1}<R_{t}$ and $y_{t}>0$. Since $y_{t}>0$, production in period $t$ covers some of the demands in periods later than $t$; therefore, one can construct another feasible production series, $\Psi_{u m^{\prime} v, r^{\prime}}^{\prime}=\left\{x_{u}^{\prime}=x_{u}, \cdots, x_{t-1}^{\prime}=x_{t-1}, x_{t}^{\prime}=R_{t}-\right.$ $\left.e, x_{t+1}^{\prime}=x_{t+1}+e, x_{t+2}^{\prime}=x_{t+2}, \cdots, x_{v-1}^{\prime}=x_{v-1}\right\}$ with $e>$ 0 . (In all of our proofs, all entities with notation (') retain their original definition and indicate recomputation for a new sequence.) Clearly, $\Psi_{u v}^{\prime}$ yields a lower cost due to the assumed marginal production cost structure. Hence, the result.

Proof of Lemma 3. We will prove by contradiction. First, suppose a feasible production series $\Psi_{u v}^{\prime}$ such that $x_{t}^{\prime}>\max \left(Q_{t},\left[D_{t}-y_{t-1}^{\prime}\right]^{+}\right), 0<x_{t+1}^{\prime}<R_{t+1}$ and $y_{t+1}^{\prime} \geq 0$. Let $q_{t}=\min \left(\left[R_{t+1}-x_{t+1}^{\prime}\right],\left[x_{t}^{\prime}-\max \left(Q_{t},\left[D_{t}-\right.\right.\right.\right.$ $\left.\left.\left.\left.y_{t-1}^{\prime}\right]^{+}\right)\right]\right)$. Then, there is a feasible series $\Psi_{u v}$ such that $x_{t}=x_{t}^{\prime}-q_{t}, x_{t+1}=x_{t+1}^{\prime}+q_{t}, y_{t+1}=y_{t+1}^{\prime}$ and $x_{i}^{\prime}=x_{i}$ for all other $i$. If $\left[R_{t+1}-x_{t+1}^{\prime}\right]<\left[x_{t}^{\prime}-\max \left(Q_{t},\left[D_{t}-y_{t-1}\right]^{+}\right)\right]$, we have $x_{t+1}=R_{t+1}$ and $x_{t}>\max \left(Q_{t},\left[D_{t}-y_{t-1}\right]^{+}\right)$; otherwise, $x_{t}=\max \left(Q_{t},\left[D_{t}-y_{t-1}\right]^{+}\right)$and $x_{t+1}<R_{t+1}$. In either case, $\Psi_{u v}$ yields a lower cost due to the assumed marginal production cost structure; therefore, $\Psi_{u v}^{\prime}$ cannot be optimal. Next, suppose a feasible production series $\Psi_{u v}^{\prime}$ such that $x_{t}^{\prime}>\max \left(Q_{t},\left[D_{t}-y_{t-1}^{\prime}\right]^{+}\right), x_{t+1}^{\prime}=R_{t+1}$ and $y_{t+1}^{\prime}=0$. This implies that $x_{t+1}^{\prime}=\left[D_{t+1}-y_{t}^{\prime}\right]^{+}=D_{t+1}$ from the demand structure that $D_{t+1} \leq R_{t+1}$. If so, $y_{t}^{\prime}=0$ and, thereby, $x_{t}^{\prime}=\left[D_{t}-y_{t-1}^{\prime}\right]^{+}$which, jointly, contradict $x_{t}^{\prime}>\max \left(Q_{t},\left[D_{t}-y_{t-1}^{\prime}\right]^{+}\right)$. Hence, the result.

Proof Theorem 2. For convenience, we will prove in reverse order.

(iii) Follows from Lemma 1 and Corollary 1 part (ii).

(ii) Note $t=m-r$. Due to the no shortages assumption, $\sum_{i=m-r}^{m} x_{i}+y_{m-r-1}=\sum_{i=m-r}^{v-1} D_{i}$. From part (iii) we have $x_{i}=R_{i}$ for $m-r+1 \leq i \leq m$. Hence, $x_{m-r}=$ $\sum_{i=m-r}^{v-1} D_{i}-y_{m-k-1}-\sum_{i=m-r+1}^{m} R_{i}$. Invoking Lemma 1, we have the result.

(i) $u \leq t \leq m-r-1$. By definition $t$ is an intermediate period; therefore, $x_{t} \nless \max \left(Q_{t},\left[D_{t}-y_{t-1}\right]^{+}\right)$. First consider $t=m-r-1$. From part (ii), $x_{t+1}=\varepsilon<R_{t+1}$. Since $x_{t+1} \neq R_{t+1}$, from Lemma $3 x_{t} \ngtr \max \left(Q_{t},\left[D_{t}-y_{t-1}\right]^{+}\right)$. Hence, the result. Next consider $t=m-r-2$. If $x_{t+1}<$ $R_{t+1}$, the arguments for period $m-r-1$ also hold. If, however, $x_{t+1}=R_{t+1}$, from Corollary 1 part (i) $y_{t+1}=0$, since $x_{t+2}=\varepsilon$ (from Theorem 2 part (ii) with $t+2=m-r$ ). Since $x_{t+1}=R_{t+1}$ and $y_{t+1}=0$, from Lemma $3, x_{t} \ngtr$ $\max \left(Q_{t},\left[D_{t}-y_{t-1}\right]^{+}\right)$. Inductively, the argumentation can be carried out for every period until $t=u$. Hence, the result.

Proof Theorem 3. (i) First consider a feasible production sequence $S_{u v}$, in an optimal plan, consisting of two series such that $S_{u v}=\Psi_{u \tau-1 \mid 0, y_{\tau-1}} \cup \Psi_{\tau v \mid y_{\tau-1}, 0}$. By definition, $y_{\tau-1}>0$. Furthermore, let $m_{1}$ and $m_{1}-r_{1}+1$ denote, respectively, the latest period in which production is done and the earliest period in which capacitated production is done in series $\Psi_{u \tau-1 \mid 0, y_{\tau-1}}$, and let $m_{2}$ and $m_{2}-r_{2}+1$ denote, respectively, the latest period in which production is done and the earliest period in which capacitated production is done in series $\Psi_{\tau v \mid y_{\tau-1}, 0}$. Suppose that $\Psi_{\tau v \mid y_{\tau-1}, 0}$ is an uncapacitated production series, i.e., $\sum_{i=\tau}^{m_{2}}\left(R_{i}-x_{i}\right)>0$. It is easy to show that a new series $\Psi_{\tau t_{2} \mid y_{\tau_{\tau-1}, 0}^{\prime}}^{\prime}$ constructed such that $x_{m_{2}-r_{2}}^{\prime}=x_{m_{2}-r_{2}}+\epsilon$ and $y_{\tau-1}^{\prime}=y_{\tau-1}-\epsilon$ while keeping everything the same improves the cost; hence, $S_{u v}$ cannot be optimal. One can carry on this construction until either $\sum_{i=\tau}^{m_{2}}\left(R_{i}-x_{i}^{\prime}\right)=0$ or $y_{\tau-1}^{\prime}=0$.

If with the new series $\Psi_{\tau v \mid y_{\tau-1}^{\prime}, 0}^{\prime}$, we end up with $y_{\tau-1}^{\prime}>0$ under $\sum_{i=\tau}^{m_{2}}\left(R_{i}-x_{i}^{\prime}\right)=0$, then, the sequence consists of one non-capacitated and one capacitated series. If we end up with $y_{\tau-1}^{\prime}=0$ under $\sum_{i=\tau}^{m_{2}}\left(R_{i}-x_{i}^{\prime}\right)=0$, then period $\tau$ is a 
regeneration period which implies that there are two production sequences such as $S_{u \tau}^{\prime}$ and $S_{\tau v}^{\prime}$, and each of these sequences consists of one series where $\Psi_{u \tau-1 \mid 0,0}^{\prime}$ is uncapacitated production series and $\Psi_{\tau v \mid 0,0}^{\prime}$ is capacity-constrained production series. A similar conclusion is drawn if $y_{\tau-1}^{\prime}=0$ but $\sum_{i=\tau}^{m_{2}}\left(R_{i}-x_{i}^{\prime}\right) \neq 0$ under the new construction of series, as well, in which both of the series are uncapacitated. Hence, a production sequence which comprises of two uncapacitated series cannot be optimal.

For the production sequences that comprises more than two series, we can apply the above argument to the last uncapacitated production series and the immediate preceeding series.

Hence, the result.

(ii) Proof follows immediately from the new series construction described in part (i).

Proof of Corollary 4. Follows from Proposition 2.1 in Bitran and Yanasse (1982) when $z_{t}=1$.

Proof of Theorem 4. (i) We will prove by contradiction. Consider the series $\Psi_{u v \mid y_{u-1}, y_{v-1}}$ with $y_{u-1}>0$ and $y_{v-1}=0$. By choosing $y_{v-1}=0$, we start our analysis with the last series in a production sequence by definition. From Theorem 3 this series is a capacitated production series due to the positive beginning inventory, $y_{u-1}$. Let $m$ be the latest period in which production is performed in the series and, let $j$ be the latest production period (in an earlier series) before $u$. Consider constructing another series $\Psi_{u v \mid y_{u-1}-\epsilon, y_{v-1}}^{\prime}$, i.e., producing $\epsilon(>0)$ units in period $m+1$ and decreasing the initial inventory in period $u$ by the same amount. The newly constructed series results in a cost difference (old cost minus new cost $)$ of at least $\left(\left[c_{j}+\sum_{i=t}^{m} h_{i}-c_{m+1}\right] \epsilon-k_{m+1}\right)$. (Note that this difference is only a conservative lower bound because producing $\epsilon$ units less in period $j$ may also result in further cost reductions due to changes within that production series.) Since $k_{t}=0$ for all $t$, due to the assumed cost structure, the difference is positive implying that the change in the series structure yields a lower cost. Hence, the original series cannot be optimal. In a similar fashion, one can continue the cost reduction until one gets $y_{u-1}=0$. If there are production series prior to the one under consideration in the sequence in which the initial inventory level is positive, starting with the last one of such series, we apply the same argument to them, until we end up with all series in the original sequence with zero beginning and ending inventories. Hence, in an optimal plan, if there is a cold setup in period $t$, i.e., $\left(z_{t} \times x_{t}>0\right)$, then $y_{t-1}=0$; thus, every period in which a cold setup is done is a regeneration point. Thus, every series is a sequence. Moreover, when $k_{m+1}=0, E_{m}$ in Lemma 1 reduces to ( $(\max$ $\left.\left(Q_{m},\left[D_{m}-y_{t-1}\right]^{+}\right)\left(c_{m}+h_{m}-c_{m+1}-\omega_{m}\right)+R_{m} \cdot \omega_{m}\right) /\left(c_{m}+\right.$ $\left.h_{m}-c_{m+1}\right)$ ) which implies that $E_{m} \leq R_{m}$. From Corollary 1 part (iii) there is no capacitated production in the given series, and the production schedule is the one given in Corollary 3 part (ii). Hence, the remainder of the result.

(ii) Follows immediately from Theorem 1 since we have established above that at a cold setup period, $y_{t-1}=0$.

Proof of Theorem 5. (i) If the demand in period $t$ is positive, the solution to the $t$-period problem implies that a setup be done in that period to satisfy the demand. Since $x_{t-1}<Q_{t-1}$, the setup will be a cold one. If the demand in period $t$ is zero, then there will be no production due to the marginal production cost structure over the horizon. In either case, the production decision is independent of the decisions in periods 1 through $t-1$. Hence, the proposed result.

(ii) The solution to the $t$-period problem implies that production in period $t$ be done with a warm setup. From $y_{t-1}=0$, we have that the demand in period $t$ must be supplied by the production in period $t$. Furthermore, from Corollary 1 part (iii), $E_{t}<R_{t}$ implies that demands in the future for longer horizons can, at the earliest, be supplied by the production in period $t$. Hence, a longer problem can be partitioned at period $t$ as proposed. (Note that if the warm setup condition is eliminated, it is easy to design problems where the production plan in periods prior to $t$ may change as the horizon of the problem is extended, and, thereby, part (ii) no longer holds.)

\section{Biographies}

Ayhan Özgür Toy received a B.Sc. in Operations Analysis from the Turkish Naval Academy, Turkey (1990), an M.Sc. in Operations Research from the Naval Postgraduate School, CA (1996) and a Ph.D. in Business Administration from Bilkent University, Turkey (2005). Currently he is a military scientist in the Turkish Navy Operations Research Division. His research interests lie in supply chain management, inventory systems and quality control.

Emre Berk received his B.Sc. and M.Sc. degrees in Mechanical Engineering from Bogazici University, Turkey (1986) and Washington State University, WA, USA (1989), respectively. He received his M.B.A. (1995) and Ph.D. degrees (1996) in Operations Management from the University of Washington. Currently, he is an Assistant Professor in the Faculty of Business Administration at Bilkent University. His research interests and expertise include supply chain management, revenue management, perishable/multi-echelon inventory theory, quality control, operations management applications in health care and manufacturing strategy. 